\title{
Less Conservative Stability Criteria for Neutral Type Neural Networks with Mixed Time-Varying Delays
}

\author{
Kaibo Shi, ${ }^{1}$ Hong Zhu, ${ }^{1}$ Shouming Zhong, ${ }^{2,3}$ Yong Zeng, ${ }^{1}$ Yuping Zhang, ${ }^{1}$ and Li Liang ${ }^{4}$ \\ ${ }^{1}$ School of Automation Engineering, University of Electronic Science and Technology of China, Chengdu 611731, China \\ ${ }^{2}$ School of Mathematical Sciences, University of Electronic Science and Technology of China, Chengdu 611731, China \\ ${ }^{3}$ Key Laboratory for Neuroinformation of Ministry of Education, University of Electronic Science and Technology of China, \\ Chengdu 611731, China \\ ${ }^{4}$ College of Information Sciences and Technology, Hainan University, Haikou 570228, China
}

Correspondence should be addressed to Kaibo Shi; skbs111@163.com

Received 20 May 2013; Accepted 7 September 2013

Academic Editor: Qiankun Song

Copyright (c) 2013 Kaibo Shi et al. This is an open access article distributed under the Creative Commons Attribution License, which permits unrestricted use, distribution, and reproduction in any medium, provided the original work is properly cited.

\begin{abstract}
This paper investigates the problem of dependent stability criteria for neutral type neural networks with mixed time-varying delays. Firstly, some new delay-dependent stability results are obtained by employing the more general partitioning approach and generalizing the famous Jensen inequality. Secondly, based on a new type of Lyapunov-Krasovskii functional with the cross terms of variables, less conservative stability criteria are proposed in terms of linear matrix inequalities (LMIs). Furthermore, it is the first time that the idea of second-order convex combination and the property of quadratic convex function applied to the derivation of neutral type neural networks play an important role in reducing the conservatism of the paper. Finally, four numerical examples are given to show the effectiveness and the advantage of the proposed method.
\end{abstract}

\section{Introduction}

During the last two decades, delayed neural networks have drawn a great deal of attention because of their extensive applications in various scientific and technical areas, such as pattern recognition, power systems, parallel computing, signal processing, finance, associative memories, mechanics of structures, and other scientific areas [1-30]. It is well known that time delay regarded as a major cause of instability and poor performance often appears in many neural networks. Therefore, the stability analysis for delayed neural networks has been investigated extensively in recent few decades. Generally speaking, studying the dynamical behavior of delayed neural networks can be mainly classified into two types: delay-independent stability and delay-dependent stability. As is known to all, delay-dependent stability criteria are less conservative than delay-independent ones when the size of time delay is small.

On the other hand, due to the complicated dynamic properties of the neural cells in the real world, there exist many neural network models such as distributed networks, chemical reactors, and heat exchanges that cannot characterize the properties of a neural reaction process precisely. It is natural and important that these systems will contain some information about the derivative of the past state to further describe and model the dynamics of the complex neural reactions. This new type of neural networks is called neutral neural networks or neural networks of neutral type. However, many researchers have focused on the global stability of neural networks of neutral type only with constant time delay in recent years, which is very restrictive. Hence, described with neutral functional differential equations with discrete and distributed delays, these neural networks called neutral type neural networks with mixed time-varying delays have a lot of research on space. The differential expression not only defines the derivative term of the current state but also explains the derivative term of the past state. Furthermore, it is necessary to have some information about the derivative of the past state in the systems to characterize the dynamics of such complex neural reactions. Practically, neutral type phenomenon always appears in studies of automatic control, 
chemical reactors, distributed networks, dynamic process including steam and water pipes, population ecology, heat exchanges, microwave oscillators, systems of turbojet engine, lossless transmission lines, vibrating masses attached to an elastic bar, and so on. For this reason, there has been a growing research interest in the study of delayed neural networks of neutral type in the recent years. Therefore, some less conservative stability criteria for neutral type neural networks with mixed time-varying delays have been reported in recently $[25,31-35]$. Many methods have been proposed in these results to reduce the conservatism of the stability criteria, such as model transformation method, free-weighting matrix method, the method of constructing novel Lyapunov-Krasovskii functionals, delay decomposition technique, and weighting-matrix decomposition method. In [36], the authors derived some less conservative stability criteria by considering some useful terms and using freeweighting matrix technique. By considering the relationship between the time-varying delay and its lower and upper bound, the results obtained in [36] were improved in [37]. By constructing a new Lyapunov-Krasovskii functional and using free-weighting matrix method, some more less conservative criteria than those obtained in [37] were proposed in [38]. Further, the problems of stability analysis of neutral type neural networks with discrete and distributed delays have been investigated in [39]. By using a delay-partitioning approach, a new type of Lyapunov-Krasovskii functionals was constructed to obtain some less conservative stability criteria. However, time delay in [39] is not only constant delay, but also the delay-partitioning approach is equational; hence, this method has some limitations.

Motivated by this technique, it is the first attempt to investigate the integral nonuniform partitioning method to be extended for neutral type neural networks with mixed time-varying delays. In the paper, the reduced conservatism of Theorem 6 benefits from the construction of the new Lyapunov-Krasovskii functionals in (17), which contain some integral nonuniform partitioning method and triple-integral terms, which play an important role in the improvement of less conservative results. Secondly, a novel handling method is given to establish the relationship among $\int_{t-h}^{t} \dot{x}(s) S_{5} \dot{x}(s) d s$, $\int_{t-h}^{t} x^{T}(s) d s$ and $x(t-h)$, which play an important role in reducing the conservatism of stability criteria further. Furthermore, compared with previous results by using the firstorder convex combination property, our derivation makes full use of the idea of second-order convex combination and the property of quadratic convex function given in the form of a lemma without employing Jensen's inequality. Finally, four numerical examples are given to illustrate the effectiveness and the advantage of the proposed main results.

Notation 1. Notations used in this paper are fairly standard: $R^{n}$ denotes the $n$-dimensional Euclidean space, $R^{n \times m}$ is the set of all $n \times m$ dimensional matrices; $I$ denotes the identity matrix of appropriate dimensions, $T$ stands for matrix transposition, the natation $X>0$ (resp., $X \geq 0$ ), for $X \in R^{n \times n}$ means that the matrix is real symmetric positive definite (resp., positive semidefinite); $\operatorname{diag}\left\{r_{1}, r_{2}, \ldots, r_{n}\right\}$ denotes block diagonal matrix with diagonal elements $r_{i}, i=$ $1,2, \ldots, n$, the symbol $*$ represents the elements below the main diagonal of a symmetric matrix, and $\langle M\rangle_{s}$ is defined as $\langle M\rangle_{s}=(1 / 2)\left(M+M^{T}\right)$.

\section{Preliminaries}

Consider the following neural networks of neutral type with mixed time-varying delays:

$$
\begin{aligned}
\dot{z}(t)= & -W_{0} z(t)+W_{1} g(z(t))+W_{2} g(z(t-h(t))) \\
& +W_{3} \int_{t-r}^{t} g^{T}(z(s)) d s+W_{4} \dot{y}(t-h(t))+J,
\end{aligned}
$$

where $z(t)=\left[z_{1}(t), \ldots, z_{n}(t)\right]^{T} \in R^{n}$ is the neural state vector, $g(z(t))=\left[g\left(z_{1}(t)\right), \ldots, g\left(z_{n}(t)\right)\right]^{T}$ is the neuron activation function, $J=\left[J_{1}, \ldots, J_{n}\right]^{T} \in R^{n}$ is an external constant input vector, and $W_{0}=\operatorname{diag}\left\{w_{01}, \ldots, w_{0 n}\right\}>0, W_{1}, W_{2}, W_{3}$, and $W_{4}$ are the constant matrices of appropriate dimensions.

Assumption A. The time-varying delay $h(t)$ is continuous and differential function that satisfies

$$
0 \leq h(t) \leq h, \quad \dot{h}(t) \leq h_{D}<1 .
$$

Assumption B. For the constants $\delta_{i}^{+}, \delta_{i}^{-}$, the nonlinear function $g_{i}(\cdot)$ in (1) satisfies the following condition:

$$
\begin{gathered}
\delta_{i}^{-} \leq \frac{g_{i}(\alpha)-g_{i}(\beta)}{\alpha-\beta} \leq \delta_{i}^{+}, \\
\forall \alpha, \beta \in R, \quad \alpha \neq \beta, \quad i=1,2, \ldots, n .
\end{gathered}
$$

Here, we denote $\bar{\Sigma}=\operatorname{diag}\left\{\delta_{1}^{+}, \ldots, \delta_{n}^{+}\right\}, \underline{\Sigma}=\operatorname{diag}\left\{\delta_{1}^{-}, \ldots, \delta_{n}^{-}\right\}$, $\Sigma=\operatorname{diag}\left\{\max \left\{\left|\delta_{1}^{+}\right|,\left|\delta_{1}^{-}\right|\right\}, \ldots, \max \left\{\left|\delta_{n}^{+}\right|,\left|\delta_{n}^{-}\right|\right\}\right\}=\operatorname{diag}\left\{\delta_{1}, \ldots\right.$, $\left.\delta_{n}\right\}, \Sigma_{1}=\operatorname{diag}\left\{\delta_{1}^{+} \delta_{1}^{-}, \ldots, \delta_{n}^{+} \delta_{n}^{-}\right\}, \Sigma_{2}=\operatorname{diag}\left\{\left(\delta_{1}^{+}+\delta_{1}^{-}\right) / 2, \ldots\right.$, $\left.\left(\delta_{n}^{+}+\delta_{n}^{-}\right) / 2\right\}$.

Assumption C. For given positive scalars $\rho_{i}$ satisfies:

$$
0=\rho_{0}<\rho_{1}<\cdots<\rho_{l}=1 \quad(i=1, \ldots, l) .
$$

It is clear that under Assumption B, the system (1) has one equilibrium point denoted as $z^{*}=\left[z_{1}^{*}, \ldots, z_{n}^{*}\right]^{T}$. For convenience, we firstly shift the equilibrium point $z^{*}$ to the origin by letting $x(t)=z(t)-z^{*}, f(x(t))=g(z(t))-g\left(z^{*}\right)$; then the system (1) can be transformed into

$$
\begin{aligned}
\dot{x}(t)= & -W_{0} x(t)+W_{1} f(x(t))+W_{2} f(x(t-h(t))) \\
& +W_{3} \int_{t-r}^{t} f^{T}(x(s)) d s+W_{4} \dot{x}(t-h(t)),
\end{aligned}
$$

where $x(t)=\left[x_{1}(t), \ldots, x_{n}(t)\right] \in R^{n}$ is the state vector of transformed system, $f(x(t))=\left[f_{1}\left(x_{1}\right), \ldots, f_{n}\left(x_{n}\right)\right]^{T} \in R^{n}$. It is easy to check that the transformed neuron activation function $f_{i}(\cdot)$ satisfies

$$
\delta_{i}^{-} \leq \frac{f_{i}(\alpha)}{\alpha} \leq \delta_{i}^{+}, \quad f_{i}(0)=0,
$$$$
\forall \alpha \in R, \quad \alpha \neq 0, \quad i=1,2, \ldots, n .
$$ 
The following lemmas are introduced, which will be used in the proof of the main results.

Fact 1 (Boyd et al. [40], (Schur complement)). For a given symmetric matrix $X=X^{T}=\left[\begin{array}{cc}X_{11} & X_{12} \\ * & X_{22}\end{array}\right]$, where $S_{11} \in R^{n \times n}$, the following conditions are equivalent:

(1) $X<0$;

(2) $X_{11}<0, X_{22}-X_{12}^{T} X_{11}^{-1} X_{12}<0$;

(3) $X_{22}<0, X_{11}-X_{12} X_{22}^{-1} X_{12}^{T}<0$.

Lemma 1 (see [41]). For symmetric matrices $Y_{0}, Y_{1}, Y_{2}$, and a vector $\xi_{t}$, Let $f(\alpha)=\alpha^{2} \xi_{t}^{T} Y_{2} \xi_{t}+\alpha \xi_{t}^{T} Y_{1} \xi_{t}+\xi_{t}^{T} Y_{0} \xi_{t}$ with $Y_{2} \leq 0$. Then we have $f\left(\alpha_{1}\right)<0$ and $f\left(\alpha_{2}\right)<0 \Rightarrow f(\alpha)<0, \forall \alpha \in$ $\left[\alpha_{1}, \alpha_{2}\right]$.

Lemma 2 (see [42]). Let $W>0$, and let $\omega(s)$ be an appropriate dimensional vector. Then, we have the following facts for any scalar function $\beta(s) \geq 0 \quad \forall s \in[a, b]$ :

$$
\begin{aligned}
& (1)-\int_{a}^{b} \omega^{T}(s) W \omega(s) d s \leq(b-a) \xi_{t}^{T} F_{1} W^{-1} F_{1} \xi_{t}+ \\
& 2 \xi_{t}^{T} F_{1} \int_{a}^{b} \omega(s) d s ;
\end{aligned}
$$

(2) $-\int_{a}^{b} \beta(s) \omega^{T}(s) W \omega(s) d s \leq \int_{a}^{b} \beta(s) d s \xi_{t}^{T} F_{2} W^{-1} F_{2} \xi_{t}+$ $2 \xi_{t}^{T} F_{2} \int_{a}^{b} \beta(s) \omega(s) d s ;$

(3) $-\int_{a}^{b} \beta^{2}(s) \omega^{T}(s) W \omega(s) d s \leq(b-a) \xi_{t}^{T} F_{3} W^{-1} F_{3} \xi_{t}+$ $2 \xi_{t}^{T} F_{3} \int_{a}^{b} \beta(s) \omega(s) d s$,

where matrices $F_{i}(i=1,2,3)$ and a vector $\xi_{t}$ independent of the integral variable are appropriate dimensional arbitrary ones.

Lemma 3 (see [43]). For any constant matrix $0<R=R^{T} \in$ $R^{n \times n}$, a scalar $r>0$ and a vector function $x:[0, r] \rightarrow R^{n}$ such that the integrations concerned are well defined; then

$$
\begin{aligned}
& -\int_{t-r}^{0} x^{T}(s) R x(s) d s \\
& \quad \leq-\frac{1}{r}\left(\int_{t-r}^{t} x(s) d s\right)^{T} R\left(\int_{t-r}^{t} x(s) d s\right), \\
& -\int_{-r}^{0} \int_{t+\theta}^{t} x^{T}(s) R x(s) d s d \theta \\
& \quad \leq-\frac{2}{r^{2}}\left(\int_{-r}^{0} \int_{t+\theta}^{t} x(s) d s d \theta\right)^{T} R\left(\int_{-r}^{0} \int_{t+\theta}^{t} x(s) d s d \theta\right) .
\end{aligned}
$$

Lemma 4 (see [44]). For any constant matrix $R \in R^{n \times n}, R=$ $R^{T}>0$, a scalar function $h:=h(t)>0$, and a vector-valued function $\dot{x}:[-h, 0] \rightarrow R^{n}$ such that the following integrations are well defined:

$$
\begin{gathered}
-h \int_{t-h}^{t} \dot{x}(s) R \dot{x}(s) d s \leq\left[\begin{array}{c}
x(t) \\
x(t-h)
\end{array}\right]^{T}\left[\begin{array}{cc}
-R & R \\
R & -R
\end{array}\right]\left[\begin{array}{c}
x(t) \\
x(t-h)
\end{array}\right], \\
-\frac{h^{2}}{2} \int_{-h}^{0} \int_{t+\theta}^{t} \dot{x}^{T}(s) R \dot{x}(s) d s d \theta \\
\leq\left[\begin{array}{c}
h x(t) \\
\int_{t-h}^{t} x(s) d s
\end{array}\right]^{T}\left[\begin{array}{cc}
-R & R \\
R & -R
\end{array}\right]\left[\begin{array}{c}
h x(t) \\
\int_{t-h}^{t} x(s) d s
\end{array}\right] .
\end{gathered}
$$

Lemma 5. Let $x(t) \in R^{n}$ has continuous derived function $\dot{x}(t)$ on interval $[0, h]$. Then for any matrix $Z^{n \times n}>0$, scalar $h>0$, the following inequality holds:

$$
\begin{aligned}
& -h \int_{t-h}^{t} \dot{x}(s) R \dot{x}(s) d s \\
& \quad \leq-\frac{2}{h}\left[\begin{array}{c}
\frac{1}{h} \int_{t-h}^{t} x(s) d s \\
x(t-h)
\end{array}\right]^{T}\left[\begin{array}{cc}
R & -R \\
-R & R
\end{array}\right]\left[\begin{array}{c}
\frac{1}{h} \int_{t-h}^{t} x(s) d s \\
x(t-h)
\end{array}\right] .
\end{aligned}
$$

Proof. From Lemma 3, we can get

$$
\begin{gathered}
\frac{1}{h}\left(\int_{t-h}^{t} \int_{t-h}^{\theta} \dot{x}(s) d s d \theta\right)^{T} R\left(\int_{t-h}^{t} \int_{t-h}^{\theta} \dot{x}(s) d s d \theta\right) \\
\quad \leq \int_{t-h}^{t}\left(\int_{t-h}^{\theta} \dot{x}(s) d s\right)^{T} R\left(\int_{t-h}^{\theta} \dot{x}(s) d s\right) d \theta \\
\quad \leq \int_{t-h}^{t} \int_{t-h}^{\theta}(\theta-(t-h)) \dot{x}^{T}(s) R \dot{x}(s) d s d \theta \\
\quad \leq \int_{t-h}^{t} \int_{s}^{t}(\theta-(t-h)) \dot{x}^{T}(s) R \dot{x}(s) d \theta d s \\
\quad=\int_{t-h}^{t}\left(\frac{h^{2}}{2}-\frac{(s-t+h)^{2}}{2}\right) \dot{x}^{T}(s) R \dot{x}(s) d s \\
\quad \leq \frac{h^{2}}{2} \int_{t-h}^{t} \dot{x}^{T}(s) R \dot{x}(s) d s .
\end{gathered}
$$

Notice that

$$
\begin{array}{r}
\left(\int_{t-h}^{t} \int_{t-h}^{\theta} \dot{x}(s) d s d \theta\right)^{T} R\left(\int_{t-h}^{t} \int_{t-h}^{\theta} \dot{x}(s) d s d \theta\right) \\
=\left[\begin{array}{c}
\int_{t-h}^{t} x(s) d s \\
h x(t-h)
\end{array}\right]^{T}\left[\begin{array}{cc}
R & -R \\
-R & R
\end{array}\right]\left[\begin{array}{c}
t \\
\int_{t-h} x(s) d s \\
h x(t-h)
\end{array}\right] .
\end{array}
$$


Therefore, we get

$$
\begin{aligned}
& -\int_{t-h}^{t} \dot{x}(s) R \dot{x}(s) d s \\
& \quad \leq-\frac{2}{h}\left[\begin{array}{c}
\frac{1}{h} \int_{t-h}^{t} x(s) d s \\
x(t-h)
\end{array}\right]^{T}\left[\begin{array}{cc}
R & -R \\
-R & R
\end{array}\right]\left[\begin{array}{c}
\frac{1}{h} \int_{t-h}^{t} x(s) d s \\
x(t-h)
\end{array}\right] .
\end{aligned}
$$

This completes the proof.

\section{Main Results}

In this section we will give sufficient conditions under which the system (5) is asymptotically stable.

Theorem 6. For given scalars $h>0$ and $h_{D}<1$, the system (5) with the neuron activation function $f(x(t))$ satisfying the condition (6) is asymptotically stable if there exists $P>0$, $R_{i}^{T}=R_{i}>0(i=1,2,3), Q_{i}^{T}=Q_{i}>0(i=1,2), S_{i}^{T}=S_{i}>$ $0(i=1, \ldots, 8), Z_{i}^{T}=Z_{i}>0(i=1, \ldots, l)$, diagonal matrices $G_{i}=\operatorname{diag}\left\{g_{i 1}, g_{2}, \ldots, g_{i n}\right\}>0, K_{i}=\operatorname{diag}\left\{k_{i 1}, k_{2}, \ldots, k_{i n}\right\}>$ $0, L_{i}=\operatorname{diag}\left\{l_{i 1}, l_{2}, \ldots, l_{i n}\right\}>0,(i=1,2,3)$ and $M=$ $\operatorname{diag}\left\{m_{i 1}, m_{i 2}, \ldots, m_{\text {in }}\right\}>0,(i=1,2,3,4), T_{i}(i=1, \ldots, 5)$ and $F_{i}(i=1, \ldots, 6)$ with appropriate dimensions such that the following symmetric linear matrix inequality holds:

$$
\begin{gathered}
\Xi_{1}=\left[\begin{array}{cccc}
\Pi_{0} & h F_{1}^{T} & h F_{2}^{T} & h F_{3}^{T} \\
* & -h R_{3} & 0 & 0 \\
* & * & -Q_{1} & 0 \\
* & * & * & -\frac{h}{3} Q_{2}
\end{array}\right]<0, \\
\Xi_{2}=\left[\begin{array}{cccc}
\Pi_{0}+h \Pi_{1} & h F_{4}^{T} & h F_{5}^{T} & h F_{6}^{T} \\
* & -h R_{3} & 0 & 0 \\
* & * & -Q_{1} & 0 \\
* & * & * & -\frac{h}{3} Q_{2}
\end{array}\right]<0,
\end{gathered}
$$

where

$$
\begin{aligned}
& \Pi_{0}=\left\langle 2\left[\begin{array}{ll}
e_{1} & e_{7}+e_{8}
\end{array}\right] P\left[\begin{array}{ll}
e_{2} & e_{1}-e_{4}
\end{array}\right]^{T}\right. \\
& +2\left[\begin{array}{ll}
e_{2} & 0
\end{array}\right] R_{1}\left[\begin{array}{ll}
0 & e_{7}+e_{8}
\end{array}\right]^{T} \\
& \left.+2\left[\begin{array}{ll}
e_{2} & 0
\end{array}\right] R_{2}\left[\begin{array}{ll}
h e_{1} & e_{8}
\end{array}\right]^{T}\right\rangle_{s}+\left[\begin{array}{ll}
e_{1} & e_{1}
\end{array}\right] \\
& \times\left(R_{1}+R_{2}\right)\left[\begin{array}{ll}
e_{1} & e_{1}
\end{array}\right]^{T} \\
& -\left(1-h_{D}\right)\left[\begin{array}{ll}
e_{1} & e_{3}
\end{array}\right] R_{1}\left[\begin{array}{ll}
e_{1} & e_{3}
\end{array}\right]^{T} \\
& -\left[\begin{array}{ll}
e_{1} & e_{4}
\end{array}\right] R_{2}\left[\begin{array}{ll}
e_{1} & e_{4}
\end{array}\right]^{T}+h\left[\begin{array}{ll}
e_{1} & e_{2}
\end{array}\right] R_{3} \\
& \times\left[\begin{array}{ll}
e_{1} & e_{2}
\end{array}\right]^{T}+e_{2}\left(h^{2} Q_{1}+h^{3} Q_{2}\right) e_{2}^{T}
\end{aligned}
$$

$$
\begin{aligned}
& +\left\langle 2 F_{1}^{T}\left[e_{7} e_{3}-e_{4}\right]^{T}+4 F_{2}^{T}\left[h e_{3}-e_{7}\right]^{T}\right. \\
& +6 F_{3}^{T}\left[h e_{3}-e_{7}\right]^{T} \\
& \left.+2 F_{4}^{T}\left[\begin{array}{ll}
e_{8} & e_{1}-e_{3}
\end{array}\right]^{T}-4 F_{5}^{T} e_{8}^{T}-6 F_{6}^{T} e_{8}^{T}\right\rangle_{s} \\
& +e_{9}\left(S_{1}+S_{2}\right) e_{9}^{T}-\left(1-h_{D}\right) e_{10} S_{1} e_{10}^{T}-e_{11} S_{2} e_{11}^{T} \\
& -e_{6} S_{4} e_{6}^{T}+e_{2}\left(S_{3}+S_{4}\right) e_{2}^{T}-\left(1-h_{D}\right) e_{5} S_{3} e_{5}^{T} \\
& +e_{2}\left(h S_{5}+\frac{h^{2}}{2} S_{6}\right) e_{2}^{T}-2 e_{1} S_{6} e_{1}^{T}+\frac{4}{h} e_{1} S_{6} e_{7}^{T} \\
& +\frac{4}{h} e_{1} S_{6} e_{8}^{T}+\frac{4}{h^{2}} e_{4} S_{5} e_{7}^{T}+\frac{4}{h^{2}} e_{4} S_{5} e_{8}^{T}-\frac{2}{h} e_{4} S_{5} e_{4}^{T} \\
& -e_{7}\left(\frac{2}{h^{3}} S_{5}+\frac{2}{h^{2}} S_{6}\right) e_{7}^{T}-e_{7}\left(\frac{4}{h^{3}} S_{5}+\frac{4}{h^{2}} S_{6}\right) e_{8}^{T} \\
& -e_{8}\left(\frac{2}{h^{3}} S_{5}+\frac{2}{h^{2}} S_{6}\right) e_{8}^{T} \\
& +e_{9}\left(r S_{7}+\frac{r^{2}}{2} S_{8}+\sum_{i=1}^{l}\left(\rho_{i}-\rho_{i-1}\right) r Z_{i}\right) e_{9}^{T} \\
& -\sum_{i=1}^{l} \frac{1}{\left(\rho_{i}-\rho_{i-1}\right) r} e_{11+i} Z_{i} e_{11+i}^{T} \\
& -\frac{1}{r}\left(e_{12}+\cdots+e_{11+l}\right) S_{7}\left(e_{12}+\cdots+e_{11+l}\right)^{T} \\
& -\frac{2}{r^{2}} e_{12+l} S_{8} e_{12+l}^{T} \\
& +e_{1}\left(\bar{\Sigma} K_{1}-\underline{\Sigma} G_{1}+L_{1} \Sigma\right) e_{2}^{T}+2 e_{2}\left(G_{1}-K_{1}+L_{1}\right) e_{9}^{T} \\
& +2 e_{3}\left(\bar{\Sigma} K_{2}-\underline{\Sigma} G_{2}+L_{2} \Sigma\right) e_{5}^{T}+2 e_{5}\left(G_{2}-K_{2}+L_{2}\right) e_{10}^{T} \\
& +2 e_{4}\left(\bar{\Sigma} K_{3}-\underline{\Sigma} G_{3}+L_{3} \Sigma\right) e_{6}^{T}+2 e_{6}\left(G_{3}-K_{3}+L_{3}\right) e_{11}^{T} \\
& +e_{1}\left(\Sigma M_{1} \Sigma-M_{2} \Sigma_{1}\right) e_{1}^{T}+2 e_{1} M_{2} \Sigma_{2} e_{9}^{T}-e_{3} M_{3} \Sigma_{1} e_{3}^{T} \\
& +2 e_{3} M_{3} \Sigma_{2} e_{10}^{T}-e_{9}\left(M_{1}+M_{2}\right) e_{9}^{T} \\
& -e_{10} M_{3} e_{10}^{T}-e_{4} M_{4} \Sigma_{1} e_{4}^{T} \\
& +2 e_{4} M_{4} \Sigma_{2} e_{11}^{T}+e_{2}\left(-T_{1}-T_{1}^{T}\right) e_{2}^{T} \\
& -e_{11} M_{4} e_{11}^{T}-2 e_{1} W_{0}^{T} T_{1}^{T} e_{2}^{T} \\
& +2 e_{2} T_{1} W_{1} e_{9}^{T}+2 e_{2} T_{1} W_{2} e_{10}^{T} \\
& +2 e_{2} T_{1} W_{3}\left(e_{12}+\cdots+e_{11+l}\right)^{T} \\
& +2 e_{2} T_{1} W_{4} e_{5}^{T}-2 e_{1} T_{2} e_{2}^{T}+e_{1}\left(-T_{2} W_{0}-W_{0}^{T} T_{2}^{T}\right) e_{1}^{T} \\
& +2 e_{1} T_{2} W_{1} e_{9}^{T}+2 e_{1} T_{2} W_{2} e_{10}^{T} \\
& +2 e_{1} T_{2} W_{3}\left(e_{12}+\cdots+e_{11+l}\right)^{T} \\
& +2 e_{2} T_{2} W_{4} e_{5}^{T}-2 e_{2} T_{3}^{T} e_{9}^{T}
\end{aligned}
$$




$$
\begin{aligned}
& -2 e_{1} W_{0}^{T} T_{3}^{T} e_{9}^{T}+2 e_{9} T_{3} W_{2} e_{10}^{T} \\
+ & e_{9}\left(T_{3} W_{1}+W_{1}^{T} T_{3}^{T}\right) e_{9}^{T}+2 e_{9} T_{3} W_{3}\left(e_{12}+\cdots+e_{11+l}\right)^{T} \\
+ & 2 e_{5} W_{4}^{T} T_{3}^{T} e_{9}^{T}-2 e_{2} T_{4}^{T} e_{10}^{T}+e_{9} W_{1}^{T} T_{4}^{T} e_{10}^{T} \\
+ & e_{10}\left(T_{4} W_{2}+W_{2}^{T} T_{4}^{T}\right) e_{10}^{T} \\
+ & 2 e_{10} T_{4} W_{3}\left(e_{12}+\cdots+e_{11+l}\right)^{T} \\
+ & 2 e_{5} W_{4}^{T} T_{4}^{T} e_{10}^{T}+2 e_{5} W_{4}^{T} T_{3}^{T} e_{9}^{T} \\
& -2 e_{2} T_{5}^{T} e_{5}^{T}-2 e_{1} W_{0}^{T} T_{5}^{T} e_{5}^{T} \\
+ & e_{5} T_{5} W_{1} e_{9}^{T}+e_{5} T_{5} W_{2} e_{10}^{T}+2 e_{5}\left(T_{5} W_{4}+W_{4}^{T} T_{4}^{T}\right) e_{5}^{T} \\
+ & 2 e_{5} T_{5} W_{3}\left(e_{12}+\cdots+e_{11+l}\right)^{T}-2 e_{1} W_{0}^{T} T_{4}^{T} e_{10}^{T}, \\
\Pi_{1}= & \left\langle 2\left(e_{2} \quad 0\right) R_{1}\left(e_{1} e_{0}\right)^{T}-4 F_{2}^{T} e_{3}^{T}-6 F_{3}^{T} e_{3}^{T}\right. \\
& \left.+4 F_{5}^{T} e_{1}^{T}+6 F_{6}^{T} e_{1}^{T}\right\rangle_{s},
\end{aligned}
$$

with

$$
\begin{array}{r}
\langle\cdot\rangle_{s}=\frac{1}{2}\left[(\cdot)+(\cdot)^{T}\right], \quad e_{i}=\left[\begin{array}{lll}
0_{n \times(i-1)} & I_{n \times n} & 0_{n \times(12+l-i)}
\end{array}\right]^{T} \\
(i=1, \ldots, 12+l) .
\end{array}
$$

Proof. Consider a novel augmented Lyapunov-Krasovskii functional for the system (5) as follows:

$$
\begin{aligned}
V\left(x_{t}\right)= & V_{1}\left(x_{t}\right)+V_{2}\left(x_{t}\right)+V_{3}\left(x_{t}\right)+V_{4}\left(x_{t}\right)+V_{5}\left(x_{t}\right) \\
& +V_{6}\left(x_{t}\right)+V_{7}\left(x_{t}\right),
\end{aligned}
$$

where

$$
\begin{gathered}
V_{1}\left(x_{t}\right)=\xi_{1}^{T}(t) P \xi_{1}(t), \\
V_{2}\left(x_{t}\right)=\int_{t-h(t)}^{t} \xi_{2}^{T}(t, s)\left\{\left[E_{1} E_{2}\right] R_{1}\left[E_{1} E_{2}\right]^{T}\right\} \xi_{2}(t, s) d s \\
+\int_{t-h}^{t} \xi_{2}^{T}(t, s)\left\{\left[E_{1} E_{2}\right] R_{2}\left[E_{1} E_{2}\right]^{T}\right\} \xi_{2}(t, s) d s,
\end{gathered}
$$

$$
\begin{aligned}
V_{3}( & \left.x_{t}\right) \\
= & \int_{t-h}^{t}(h-t+s) \xi_{2}^{T}(t, s)\left\{\left[E_{2} E_{3}\right] R_{3}\left[E_{2} E_{3}\right]^{T}\right\} \xi_{2}(t, s) d s \\
& +\int_{t-h}^{t}(h-t+s)^{2} \xi_{2}^{T}(t, s)\left\{E_{3} Q_{1} E_{3}^{T}\right\} \xi_{2}(t, s) d s \\
& +\int_{t-h}^{t}(h-t+s)^{3} \xi_{2}^{T}(t, s)\left\{E_{3} Q_{2} E_{3}^{T}\right\} \xi_{2}(t, s) d s,
\end{aligned}
$$

$$
\begin{aligned}
& V_{4}\left(x_{t}\right) \\
& =\int_{t-h(t)}^{t} f^{T}(x(s)) S_{1} f(x(s)) d s \\
& +\int_{t-h}^{t} f^{T}(x(s)) S_{2} f(x(s)) d s \\
& +\int_{t-h(t)}^{t} \dot{x}^{T}(s) S_{3} \dot{x}(s) d s \\
& +\int_{t-h}^{t} \dot{x}^{T}(s) S_{4} \dot{x}(s) d s, \\
& V_{5}\left(x_{t}\right)=\int_{t-h}^{t}(h-t+s) \dot{x}^{T}(s) S_{5} \dot{x}(s) d s \\
& +\int_{-h}^{0} \int_{\theta}^{0} \int_{t+\lambda}^{t} \dot{x}^{T}(s) S_{6} \dot{x}(s) d s d \lambda d \theta, \\
& V_{6}\left(x_{t}\right)=\int_{t-r}^{t}(r-t+s) f^{T}(x(s)) S_{7} f(x(s)) d s \\
& +\int_{-r}^{0} \int_{\theta}^{0} \int_{t+\lambda}^{t} f^{T}(x(s)) S_{8} f(x(s)) d s d \lambda d \theta \\
& +2 \sum_{i=1}^{l} \int_{-\rho_{i} r}^{-\rho_{i-1} r} \int_{t+\theta}^{t} f(x(s)) Z_{i} f(x(s)) d s d \theta, \\
& V_{7}\left(x_{t}\right)=2 \sum_{i=1}^{n} g_{1 i} \int_{0}^{x_{i}(t)}\left(f_{i}(s)-\delta_{i}^{-} s\right) d s \\
& +2 \sum_{i=1}^{n} k_{1 i} \int_{0}^{x_{i}(t)}\left(\delta_{i}^{+} s-f_{i}(s)\right) d s \\
& +2 \sum_{i=1}^{n} l_{1 i} \int_{0}^{x_{i}(t)}\left(f_{i}(s)+\delta_{i} s\right) d s \\
& +2 \sum_{i=1}^{n} g_{2 i} \int_{0}^{x_{i}(t-h(t))}\left(f_{i}(s)-\delta_{i}^{-} s\right) d s \\
& +2 \sum_{i=1}^{n} k_{2 i} \int_{0}^{x_{i}(t-h(t))}\left(\delta_{i}^{+} s-f_{i}(s)\right) d s \\
& +2 \sum_{i=1}^{n} l_{2 i} \int_{0}^{x_{i}(t-h(t))}\left(f_{i}(s)+\delta_{i} s\right) d s \\
& +2 \sum_{i=1}^{n} g_{3 i} \int_{0}^{x_{i}(t-h)}\left(f_{i}(s)-\delta_{i}^{-} s\right) d s \\
& +2 \sum_{i=1}^{n} k_{3 i} \int_{0}^{x_{i}(t-h)}\left(\delta_{i}^{+} s-f_{i}(s)\right) d s \\
& +2 \sum_{i=1}^{n} l_{3 i} \int_{0}^{x_{i}(t-h)}\left(f_{i}(s)+\delta_{i} s\right) d s,
\end{aligned}
$$


with

$$
\begin{aligned}
& \xi_{1}^{T}(t)=\left[x^{T}(t) \int_{t-h}^{t} x^{T}(s) d s\right], \\
& \xi_{2}^{T}(t, s)=\left[\begin{array}{lll}
x^{T}(t) & x^{T}(s) & \dot{x}^{T}(s)
\end{array}\right], \\
& \xi_{3}^{T}(t)=\left[\begin{array}{llll}
x^{T}(t) & \dot{x}^{T}(t) & x^{T}(t-h(t)) & x^{T}(t-h)
\end{array}\right. \\
& \left.\dot{x}^{T}(t-h(t)) \dot{x}^{T}(t-h)\right], \\
& \xi_{4}^{T}(t)=\left[\int_{t-h}^{t-h(t)} x^{T}(s) d s \int_{t-h(t)}^{t} x^{T}(s) d s f^{T}(x(t))\right. \\
& \left.f^{T}(x(t-h(t))) \quad f^{T}(x(t-h))\right], \\
& \xi_{5}^{T}(t)=\left[\int_{t-\rho_{1} r}^{t} f^{T}(x(s)) d s \cdots \int_{t-r}^{t-\rho_{l-1} r} f^{T}(x(s)) d s\right],
\end{aligned}
$$

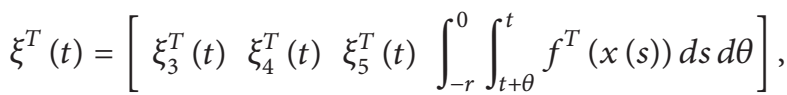

$$
\begin{aligned}
& E_{i}=\left[\begin{array}{lll}
0_{n \times(i-1)} & I_{n \times n} & 0_{n \times(3-i)}
\end{array}\right]^{T} \quad(i=1,2,3) .
\end{aligned}
$$

The time derivative of $V\left(x_{t}\right)$ along the trajectory of system (5) is given as

$$
\begin{aligned}
\dot{V}\left(x_{t}\right)= & \dot{V}_{1}\left(x_{t}\right)+\dot{V}_{2}\left(x_{t}\right)+\dot{V}_{3}\left(x_{t}\right)+\dot{V}_{4}\left(x_{t}\right) \\
& +\dot{V}_{5}\left(x_{t}\right)+\dot{V}_{6}\left(x_{t}\right)+\dot{V}_{7}\left(x_{t}\right),
\end{aligned}
$$

where

$$
\begin{aligned}
& \dot{V}_{1}\left(x_{t}\right)=2 \xi_{1}^{T}(t) P \dot{\xi}_{1}(t) \\
& =2 \xi^{T}(t)\left[\begin{array}{ll}
e_{1} & e_{7}+e_{8}
\end{array}\right] P\left[\begin{array}{ll}
e_{2} & e_{1}-e_{4}
\end{array}\right]^{T} \xi(t), \\
& \dot{V}_{2}\left(x_{t}\right) \leq\left[\begin{array}{ll}
x^{T}(t) & x^{T}(t)
\end{array}\right] R_{1}\left[\begin{array}{ll}
x^{T}(t) & x^{T}(t)
\end{array}\right]^{T} \\
& +2\left[\begin{array}{ll}
\dot{x}^{T}(t) & 0
\end{array}\right] R_{1} \int_{t-h(t)}^{t}\left[\begin{array}{ll}
x^{T}(t) & x^{T}(s)
\end{array}\right]^{T} d(s) \\
& -\left(1-h_{D}\right) \times\left[x^{T}(t) x^{T}(t-h(t))\right] R_{1} \\
& \times\left[\begin{array}{ll}
x^{T}(t) & x^{T}(t-h(t))
\end{array}\right]^{T}+\left[\begin{array}{ll}
x^{T}(t) & \left.x^{T}(t)\right] R_{2}
\end{array}\right. \\
& \times\left[\begin{array}{ll}
x^{T}(t) & x^{T}(t)
\end{array}\right]^{T}+2\left[\begin{array}{ll}
\dot{x}^{T}(t) & 0
\end{array}\right] R_{2} \\
& \times \int_{t-h}^{t}\left[\begin{array}{ll}
x^{T}(t) & x^{T}(s)
\end{array}\right]^{T} d(s)-\left[\begin{array}{ll}
x^{T}(t) & x^{T}(t-h)
\end{array}\right] \\
& \times R_{2}\left[\begin{array}{ll}
x^{T}(t) & x^{T}(t-h)
\end{array}\right]^{T}
\end{aligned}
$$$$
=\xi^{T}(t)\left\{\left(\begin{array}{ll}
e_{1} & e_{1}
\end{array}\right) R_{1}\left(\begin{array}{ll}
e_{1} & e_{1}
\end{array}\right)^{T}+2\left(\begin{array}{ll}
e_{2} & 0
\end{array}\right)\right.
$$$$
\times R_{1}\left(h(t) e_{1} e_{8}\right)^{T}-\left(1-h_{D}\right)
$$$$
\times\left(\begin{array}{ll}
e_{1} & e_{3}
\end{array}\right) R_{1}\left(\begin{array}{ll}
e_{1} & e_{3}
\end{array}\right)^{T}+\left(\begin{array}{ll}
e_{1} & e_{1}
\end{array}\right)
$$$$
\times R_{2}\left(\begin{array}{ll}
e_{1} & e_{1}
\end{array}\right)^{T}+2\left(\begin{array}{ll}
e_{2} & 0
\end{array}\right) R_{2}
$$$$
\times\left(\begin{array}{ll}
h e_{1} & e_{7}+e_{8}
\end{array}\right)^{T}-\left(\begin{array}{ll}
e_{1} & e_{4}
\end{array}\right)
$$$$
\left.\times R_{2}\left(e_{1} e_{4}\right)^{T}\right\} \xi(t),
$$$$
\dot{V}_{3}\left(x_{t}\right)=h \xi_{2}(t, t)\left\{\left[E_{2} E_{3}\right] R_{3}\left[E_{2} E_{3}\right]^{T}\right\} \xi_{2}(t, t)
$$$$
+\dot{x}^{T}(t)\left(h^{2} Q_{1}+h^{3} Q_{2}\right) \dot{x}(t)+V_{a}(x(t))
$$$$
=\xi^{T}(t)\left\{h\left(\begin{array}{ll}
e_{1} & e_{2}
\end{array}\right) R_{3}\left(\begin{array}{ll}
e_{1} & e_{2}
\end{array}\right)^{T}\right.
$$$$
\left.+e_{2}\left(h^{2} Q_{1}+h^{3} Q_{2}\right) e_{2}^{T}\right\} \xi(t)
$$$$
+V_{a}(x(t)) \text {. }
$$

Here, $V_{a}(x(t))$ is the sum of all integral terms expressed as

$$
\begin{aligned}
& V_{a}((t)) \\
&=-\int_{t-h}^{t} \eta_{2}(t, s)\left\{\left[E_{2} E_{3}\right] R_{3}\left[E_{2} E_{3}\right]^{T}\right\} \eta_{2}(t, s) d s \\
&-\int_{t-h}^{t} 2(h-t+s) \eta_{2}(t, s)\left\{E_{3} Q_{1} E_{3}^{T}\right\} \eta_{2}(t, s) d s \\
&-\int_{t-h}^{t} 3(h-t+s)^{2} \eta_{2}(t, s)\left\{E_{3} Q_{2} E_{3}^{T}\right\} \eta_{2}(t, s) d s \\
& \leq-\int_{t-h}^{t-h(t)} \eta_{2}(t, s)\left\{\left[E_{2} E_{3}\right] R_{3}\left[E_{2} E_{3}\right]^{T}\right\} \eta_{2}(t, s) d s \\
&-\int_{t-h}^{t-h(t)} 2(h-t+s) \eta_{2}(t, s)\left\{E_{3} Q_{1} E_{3}^{T}\right\} \eta_{2}(t, s) d s \\
&-\int_{t-h}^{t-h(t)} 3(h-t+s)^{2} \eta_{2}(t, s)\left\{E_{3} Q_{2} E_{3}^{T}\right\} \eta_{2}(t, s) d s \\
&-\int_{t-h(t)}^{t} \eta_{2}(t, s)\left\{\left[E_{2} E_{3}\right] R_{3}\left[E_{2} E_{3}\right]^{T}\right\} \eta_{2}(t, s) d s \\
&-\int_{t-h(t)}^{t} 2(h(t)-t+s) \eta_{2}(t, s)\left\{E_{3} Q_{1} E_{3}^{T}\right\} \eta_{2}(t, s) d s \\
&-\int_{t-h(t)}^{t} 3(h(t)-t+s)^{2} \eta_{2}(t, s)\left\{E_{3} Q_{2} E_{3}^{T}\right\} \\
&= \widehat{V}_{a}(x(t)) . \\
& \times \eta_{2}(t, s) d s
\end{aligned}
$$


Apply Lemma 2 to $\widehat{V}_{a}(x(t))$,

$$
\begin{aligned}
\widehat{V}_{a}(x(t)) \leq \xi^{T}(t)\{ & (h-h(t)) F_{1}^{T} R_{3}^{-1} F_{1}+2 F_{1}^{T}\left[\begin{array}{ll}
e_{7} & e_{3}-e_{4}
\end{array}\right]^{T} \\
& +(h-h(t))^{2} F_{2}^{T} Q_{1}^{-1} F_{2} \\
& +4 F_{2}^{T}\left[(h-h(t)) e_{3}-e_{7}\right]^{T} \\
& +3(h-h(t)) F_{3}^{T} Q_{1}^{-1} F_{3} \\
& +6 F_{3}^{T}\left[(h-h(t)) e_{3}-e_{7}\right]^{T} \\
& +h(t) F_{4}^{T} R_{3}^{-1} F_{4}+2 F_{4}^{T}\left(e_{8} e_{1}-e_{3}\right)^{T} \\
& +h^{2}(t) F_{5}^{T} Q_{1}^{-1} F_{5}+4 F_{5}^{T}\left[h(t) e_{1}-e_{8}\right]^{T} \\
& \left.+3 h(t) F_{6}^{T} Q_{2}^{-1} F_{6}+6 F_{6}^{T}\left[h(t) e_{1}-e_{8}\right]^{T}\right\} \\
\times & \xi(t), \quad \\
\dot{V}_{4}\left(x_{t}\right) \leq & f^{T}(x(t)) S_{1} f(x(t)) \\
& -\left(1-h_{D}\right) f^{T}(x(t-h(t))) S_{1} f(x(t-h(t))) \\
+ & f^{T}(x(t)) S_{2} f(x(t)) \\
& -f^{T}(x(t-h)) S_{2} f(x(t-h))+\dot{x}^{T}(t) S_{3} \dot{x}(t) \\
& -\left(1-h_{D}\right) \dot{x}^{T}(t-h(t)) S_{3} \dot{x}(t-h(t)) \\
+ & \dot{x}^{T}(t) S_{4} \dot{x}(t)-\dot{x}^{T}(t-h) S_{4} \dot{x}(t-h) \\
= & \xi^{T}(t)\left\{e_{9}\left(S_{1}+S_{2}\right) e_{9}^{T}-\left(1-h_{D}\right) e_{10} S_{1} e_{10}^{T}\right. \\
& \quad-e_{11} S_{2} e_{11}^{T}+e_{2}\left(S_{3}+S_{4}\right) e_{2}^{T} \\
& \left.\quad-\left(1-h_{D}\right) e_{5} S_{3} e_{5}^{T}-e_{6} S_{4} e_{6}^{T}\right\} \xi(t),
\end{aligned}
$$$$
\dot{V}_{5}\left(x_{t}\right)=\dot{x}^{T}(t)\left(h S_{5}+\frac{h^{2}}{2} S_{6}\right) \dot{x}(t)
$$$$
-\int_{t-h}^{t} \dot{x}^{T}(s) S_{5} \dot{x}(s) d s
$$$$
-\int_{-h}^{0} \int_{t+\theta}^{t} \dot{x}^{T}(s) S_{6} \dot{x}(s) d s d \theta \text {. }
$$

From Lemmas 4 and 5, we have

$$
\begin{aligned}
& -\int_{t-h}^{t} \dot{x}^{T}(s) S_{5} \dot{x}(s) d s \\
& \leq-\frac{2}{h}\left[\begin{array}{c}
\frac{1}{h} \int_{t-h}^{t} x(s) d s \\
x(t-h)
\end{array}\right]^{T}\left[\begin{array}{cc}
S_{5} & -S_{5} \\
-S_{5} & S_{5}
\end{array}\right] \\
& \times\left[\begin{array}{c}
\frac{1}{h} \int_{t-h}^{t} x(s) d s \\
x(t-h)
\end{array}\right], \\
& -\int_{-h}^{0} \int_{t+\theta}^{t} \dot{x}^{T}(s) S_{6} \dot{x}(s) d s d \theta \\
& \leq \frac{2}{h^{2}}\left[\begin{array}{c}
h x(t) \\
\int_{t-h}^{t} x(s) d s
\end{array}\right]^{T}\left[\begin{array}{cc}
-S_{6} & S_{6} \\
S_{6} & -S_{6}
\end{array}\right]\left[\begin{array}{c}
h x(t) \\
\int_{t-h}^{t} x(s) d s
\end{array}\right] .
\end{aligned}
$$

Then combining (29) and (30), we can obtain that $\dot{V}_{5}\left(x_{t}\right)$

$$
\begin{aligned}
\leq \xi^{T}(t)\{ & e_{2}\left(h S_{5}+\frac{h^{2}}{2} S_{6}\right) e_{2}^{T} \\
& -2 e_{1} S_{6} e_{1}^{T}+\frac{4}{h} e_{1} S_{6} e_{7}^{T}+\frac{4}{h} e_{1} S_{6} e_{8}^{T} \\
& +\frac{4}{h^{2}} e_{4} S_{5} e_{7}^{T}+\frac{4}{h^{2}}(t) e_{4} S_{5} e_{8}^{T}-\frac{2}{h} e_{4} S_{5} e_{4}^{T} \\
& -e_{7}\left(\frac{2}{h^{3}} S_{5}+\frac{2}{h^{2}} S_{6}\right) e_{7}^{T}-e_{7}\left(\frac{4}{h^{3}} S_{5}+\frac{4}{h^{2}} S_{6}\right) e_{8}^{T} \\
& \left.-e_{8}\left(\frac{2}{h^{3}} S_{5}+\frac{2}{h^{2}} S_{6}\right) e_{8}^{T}\right\} \xi(t),
\end{aligned}
$$

$$
\begin{aligned}
\dot{V}_{6}\left(x_{t}\right) & \\
= & r f^{T}(x(t)) S_{7} f(x(t)) \\
& -\int_{t-r}^{t} f^{T}(x(s)) S_{7} f(x(s)) d s+\frac{r^{2}}{2} f^{T}(x(t)) S_{8} f(x(t)) \\
& -\int_{-r}^{0} \int_{t+\theta}^{t} f(x(s)) S_{8} f(x(s)) d s d \theta \\
& +\sum_{i=1}^{l}\left(\rho_{i}-\rho_{i-1}\right) r f^{T}(x(t)) Z_{i} f(x(t)) \\
& -\sum_{i=1}^{l} \int_{t-\rho_{i} r}^{t-\rho_{i-1} r} f^{T}(x(s)) Z_{i} f(x(s)) d s .
\end{aligned}
$$

From Lemma 3, we get

$$
\begin{aligned}
& -\int_{t-r}^{t} f^{T}(x(s)) S_{7} f^{T}(x(s)) d s \\
& \leq-\frac{1}{r} \int_{t-r}^{t} f^{T}(x(s)) d s S_{7} \int_{t-r}^{t} f(x(s)) d s, \\
& -\int_{-r}^{0} \int_{t+\theta}^{t} f^{T}(x(s)) S_{8} f(x(s)) d s d \theta \\
& \leq-\frac{2}{r^{2}} \int_{-r}^{0} \int_{t+\theta}^{t} f^{T}(x(s)) d s d \theta S_{8} \\
& \quad \times \int_{-r}^{0} \int_{t+\theta}^{t} f(x(s)) d s d \theta \\
& -\int_{t-\rho_{i} r}^{t-\rho_{i-1} r} f^{T}(x(s)) Z_{i} f(x(s)) d s \\
& \leq-\frac{1}{\left(\rho_{i}-\rho_{i-1}\right) r} \int_{t-\rho_{i} r}^{t-\rho_{i-1} r} f^{T}(x(s)) d s Z_{i} \\
& \quad \times \int_{t-\rho_{i} r}^{t-\rho_{i-1} r} f(x(s)) d s .
\end{aligned}
$$


Then combining (32) and (33) we can have that

$$
\begin{aligned}
& \dot{V}_{6}\left(x_{t}\right) \leq r f^{T}(x(t)) S_{7} f(x(t))+\frac{r^{2}}{2} f^{T}(x(t)) S_{8} f(x(t)) \\
& +\sum_{i=1}^{l}\left(\rho_{i}-\rho_{i-1}\right) r f^{T}(x(t)) Z_{i} f(x(t)) \\
& -\frac{1}{r} \sum_{i=1}^{l} \int_{t-\rho_{i} r}^{t-\rho_{i-1} r} f^{T}(x(s)) d s S_{7} \sum_{i=1}^{l} \int_{t-\rho_{i} r}^{t-\rho_{i-1} r} f(x(s)) d s \\
& -\frac{2}{r^{2}} \int_{-r}^{0} \int_{t+\theta}^{t} f^{T}(x(s)) d s d \theta S_{8} \int_{-r}^{0} \int_{t+\theta}^{t} f(x(s)) d s \\
& -\sum_{i=1}^{l} \frac{1}{\left(\rho_{i}-\rho_{i-1}\right) r} \int_{t-\rho_{i} r}^{t-\rho_{i-1} r} f^{T}(x(s)) d s Z_{i} \\
& \times \int_{t-\rho_{i} r}^{t-\rho_{i-1} r} f(x(s)) d s \\
& =\xi^{T}(t)\left\{e_{9}\left(r S_{7}+\frac{r^{2}}{2} S_{8}+\sum_{i=1}^{l}\left(\rho_{i}-\rho_{i-1}\right) r Z_{i}\right) e_{9}^{T}\right. \\
& -\frac{2}{r^{2}} e_{12+l} S_{8} e_{12+l}^{T}-\sum_{i=1}^{l} \frac{1}{\left(\rho_{i}-\rho_{i-1}\right) r} e_{11+i} \\
& \times Z_{i} e_{11+i}^{T}-\frac{1}{r}\left(e_{12}+\cdots+e_{11+l}\right) \\
& \left.\times S_{7}\left(e_{12}+\cdots+e_{11+l}\right)^{T}\right\} \xi(t)
\end{aligned}
$$

$$
\begin{aligned}
\dot{V}_{7}\left(x_{t}\right) & \\
\leq 2 & {\left[f^{T}(x(t))\left(G_{1}-K_{1}+L_{1}\right)\right.} \\
& \left.+x^{T}(t)\left(\bar{\Sigma} K_{1}-\underline{\Sigma} G_{1}+L_{1} \Sigma\right)\right] \\
\times & \dot{x}(t)+2\left(1-h_{D}\right) \\
\times & {\left[f^{T}(x(t-h(t)))\left(G_{2}-K_{2}+L_{2}\right)\right.} \\
& \left.+x^{T}(t-h(t))\left(\bar{\Sigma} K_{2}-\underline{\Sigma} G_{2}+L_{2} \Sigma\right)\right] \\
& \times \dot{x}(t-h(t)) \\
+ & 2\left[f^{T}(x(t-h))\left(G_{3}-K_{3}+L_{3}\right)\right. \\
& \left.+x^{T}(t-h)\left(\bar{\Sigma} K_{3}-\underline{\Sigma}_{3}+L_{3} \Sigma\right)\right] \\
\times & \dot{x}(t-h) \\
= & 2 \xi^{T}(t)\left\{e_{1}\left(\bar{\Sigma} K_{1}-\underline{\Sigma} G_{1}+L_{1} \Sigma\right) e_{2}^{T}\right. \\
& +2 e_{2}\left(G_{1}-K_{1}+L_{1}\right) e_{9}^{T} \\
& +2 e_{3}\left(\bar{\Sigma} K_{2}-\underline{\Sigma} G_{2}+L_{2} \Sigma\right) e_{5}^{T} \\
& +2 e_{5}\left(G_{2}-K_{2}+L_{2}\right) e_{10}^{T}
\end{aligned}
$$

$$
\begin{aligned}
& +2 e_{4}\left(\bar{\Sigma} K_{3}-\underline{\Sigma} G_{3}+L_{3} \Sigma\right) e_{6}^{T} \\
& \left.+2 e_{6}\left(G_{3}-K_{3}+L_{3}\right) e_{11}^{T}\right\} \xi(t) .
\end{aligned}
$$

From (6), for any positive diagonal matrices $M_{i}=$ $\operatorname{diag}\left\{m_{i 1}, \ldots, \min \right\}(i=1,2,3,4)$ one can easily check

$$
\begin{aligned}
& 0 \leq\left[x^{T}(t) \Sigma M_{1} \sum x(t)-f^{T}(x(t)) M_{1} f(x(t))\right] \\
& +\left[-x^{T}(t) M_{2} \Sigma_{1} x(t)+2 x^{T}(t) M_{2} \Sigma_{2} f(x(t))\right. \\
& \left.-f^{T}(x(t)) M_{2} f(x(t))\right] \\
& +\left[-x^{T}(t-h(t)) M_{3}\right. \\
& \times \Sigma_{1} x(t-h(t))+2 x^{T}(t-h(t)) \\
& \times M_{3} \Sigma_{2} f(x(t-h(t)))-f^{T}(x(t-h(t))) \\
& \left.\times M_{3} f(x(t-h(t)))\right] \\
& +\left[-x^{T}(t-h) M_{4} \Sigma_{1} x(t-h)+2 x^{T}(t-h) M_{4} \Sigma_{2} f\right. \\
& \left.\times(x(t-h))-f^{T}(x(t-h)) M_{4} f(x(t-h))\right] \\
& =\xi^{T}(t)\left\{e_{1}\left(\Sigma M_{1} \Sigma-M_{2} \Sigma_{1}\right) e_{1}^{T}+2 e_{1} M_{2} \Sigma_{2} e_{9}^{T}-e_{3} M_{3} \Sigma_{1} e_{3}^{T}\right. \\
& +2 e_{3} M_{3} \Sigma_{2} e_{10}^{T}-e_{9}\left(M_{1}+M_{2}\right) e_{9}^{T} \\
& -e_{10} M_{3} e_{10}^{T}-e_{4} M_{4} \Sigma_{1} e_{4}^{T}+2 e_{4} M_{4} \Sigma_{2} e_{11}^{T} \\
& \left.-e_{11} M_{4} e_{11}^{T}\right\} \xi(t) \text {. }
\end{aligned}
$$

Furthermore, for arbitrary matrices $T_{1}, T_{2}, T_{3}, T_{4}, T_{5}$ with appropriate dimensions, we have

$$
\begin{aligned}
& 2 \dot{x}^{T}(t) T_{1} \\
& \qquad\left[-\dot{x}(t)-W_{0} x(t)+W_{1} f(x(t))\right. \\
& +W_{2} f(x(t-h(t))) \\
& \left.+W_{3} \int_{t-r}^{t} f^{T}(x(s)) d s+W_{4}(\dot{x}(t-h(t)))\right] \\
& =\xi^{T}(t)\left\{e_{2}\left(-T_{1}-T_{1}^{T}\right) e_{2}^{T}-2 e_{1} W_{0}^{T} T_{1}^{T} e_{2}^{T}\right. \\
& \quad+2 e_{2} T_{1} W_{1} e_{9}^{T}+2 e_{2} T_{1} W_{2} e_{10}^{T} \\
& \quad+2 e_{2} T_{1} W_{3}\left(e_{12}+\cdots+e_{11+l}\right)^{T} \\
& \left.\quad+2 e_{2} T_{1} W_{4} e_{5}^{T}\right\} \xi(t)=0
\end{aligned}
$$




$$
\begin{aligned}
& 2 x^{T}(t) T_{2} \\
& \times\left[-\dot{x}(t)-W_{0} x(t)+W_{1} f(x(t))+W_{2} f(x(t-h(t)))\right. \\
& \left.+W_{3} \int_{t-r}^{t} f^{T}(x(s)) d s+W_{4}(\dot{x}(t-h(t)))\right] \\
& =\xi^{T}(t)\left\{-2 e_{1} T_{2} e_{2}^{T}+e_{1}\left(-T_{2} W_{0}-W_{0}^{T} T_{2}^{T}\right) e_{1}^{T}\right. \\
& \quad+2 e_{1} T_{2} W_{1} e_{9}^{T}+2 e_{1} T_{2} W_{2} e_{10}^{T} \\
& +2 e_{1} T_{2} W_{3}\left(e_{12}+\cdots+e_{11+l}\right)^{T} \\
& \left.\quad+2 e_{2} T_{2} W_{4} e_{5}^{T}\right\} \xi(t)=0,
\end{aligned}
$$

$$
\begin{aligned}
& 2 f^{T}(x(t)) T_{3} \\
& \times\left[-\dot{x}(t)-W_{0} x(t)+W_{1} f(x(t))\right. \\
& +W_{2} f(x(t-h(t)))+W_{3} \int_{t-r}^{t} f^{T}(x(s)) d s \\
& \left.+W_{4}(\dot{x}(t-h(t)))\right] \\
& =\xi^{T}(t)\left\{-2 e_{2} T_{3}^{T} e_{9}^{T}-2 e_{1} W_{0}^{T} T_{3}^{T} e_{9}^{T}\right. \\
& \quad+e_{9}\left(T_{3} W_{1}+W_{1}^{T} T_{3}^{T}\right) e_{9}^{T}+2 e_{9} T_{3} W_{2} e_{10}^{T} \\
& \quad+2 e_{9} T_{3} W_{3}\left(e_{12}+\cdots+e_{11+l}\right)^{T} \\
& \left.\quad+2 e_{5} W_{4}^{T} T_{3}^{T} e_{9}^{T}\right\} \xi(t)=0,
\end{aligned}
$$$$
2 f^{T}(x(t-h(t))) T_{4}
$$$$
\times\left[-\dot{x}(t)-W_{0} x(t)+W_{1} f(x(t))\right.
$$$$
+W_{2} f(x(t-h(t)))
$$$$
+W_{3} \int_{t-r}^{t} f^{T}(x(s)) d s
$$$$
\left.+W_{4}(\dot{x}(t-h(t)))\right]
$$$$
=\xi^{T}(t)\left\{-2 e_{2} T_{4}^{T} e_{10}^{T}-2 e_{1} W_{0}^{T} T_{4}^{T} e_{10}^{T}\right.
$$$$
+e_{9} W_{1}^{T} T_{4}^{T} e_{10}^{T}+e_{10}\left(T_{4} W_{2}+W_{2}^{T} T_{4}^{T}\right) e_{10}^{T}
$$$$
+2 e_{10} T_{4} W_{3}\left(e_{12}+\cdots+e_{11+l}\right)^{T}
$$$$
\left.+2 e_{5} W_{4}^{T} T_{4}^{T} e_{10}^{T}\right\} \xi(t)=0,
$$

$$
\begin{aligned}
\dot{x}^{T}(t- & h(t)) T_{5} \\
\times[ & -\dot{x}(t)-W_{0} x(t)+W_{1} f(x(t)) \\
& +W_{2} f(x(t-h(t))) \\
& +W_{3} \int_{t-r}^{t} f^{T}(x(s)) d s \\
& \left.+W_{4}(\dot{x}(t-h(t)))\right]
\end{aligned}
$$

$$
\begin{aligned}
=\xi^{T}(t)\{ & -2 e_{2} T_{5}^{T} e_{5}^{T}-2 e_{1} W_{0}^{T} T_{5}^{T} e_{5}^{T} \\
& +e_{5} T_{5} W_{1} e_{9}^{T}+e_{5} T_{5} W_{2} e_{10}^{T} \\
& +2 e_{5} T_{5} W_{3}\left(e_{12}+\cdots+e_{11+l}\right)^{T} \\
& \left.+2 e_{5}\left(T_{5} W_{4}+W_{4}^{T} T_{4}^{T}\right) e_{5}^{T}\right\} \xi(t)=0 .
\end{aligned}
$$

The combination of (26)-(37) gives that

$$
V\left(x_{t}\right) \leq \xi^{T}(t)\left\{\Pi_{0}+h(t) \Pi_{1}+\Pi_{h}\right\} \xi(t),
$$

where $\Pi_{0}, \Pi_{1}$ are defined in (13) and (14), respectively, and

$$
\begin{aligned}
\Pi_{h}= & (h-h(t)) F_{1}^{T} R_{3}^{-1} F_{1} \\
& +(h-h(t))^{2} F_{2}^{T} Q_{1}^{-1} F_{2}+3(h-h(t)) F_{3}^{T} Q_{1}^{-1} F_{3} \\
& +h(t) F_{4}^{T} R_{3}^{-1} F_{4}+h^{2}(t) F_{5}^{T} Q_{1}^{-1} F_{5}+3 h(t) F_{6}^{T} Q_{2}^{-1} F_{6} .
\end{aligned}
$$

Note that the scalar valued function $\xi^{T}(t)\left\{\Pi_{0}+h(t) \Pi_{1}+\right.$ $\left.\Pi_{h}\right\} \xi(t)$ is quadratic function on the scalar $h(t)$ and the coefficient of second order is $\xi^{T}(t)\left[F_{2}^{T} Q_{1}^{-1} F_{2}+F_{5}^{T} Q_{1}^{-1} F_{5}\right] \xi(t) \geq$ 0 since $Q_{1}>0$. This means that the function $\xi^{T}(t)\left\{\Pi_{0}+\right.$ $\left.h(t) \Pi_{1}+\Pi_{h}\right\} \xi(t)$ is a convex quadratic function for $h(t)$. Finally, apply Fact 1 and Lemma 1 in order, then we get

$$
\begin{gathered}
\Xi_{1}<0 \text { in }(22), \quad \Xi_{2}<0 \text { in }(23) \\
\Longleftrightarrow\left[\Pi_{0}+h(t) \Pi_{1}+\Pi_{h}\right]_{h(t)=0}<0, \\
{\left[\Pi_{0}+h(t) \Pi_{1}+\Pi_{h}\right]_{h(t)=h}<0,} \\
\Longleftrightarrow \Pi_{0}+h(t) \Pi_{1}+\Pi_{h}<0, \quad \forall h(t) \in[0, h],
\end{gathered}
$$

which means the asymptotic stability of the system (19). This completes the proof.

Remark 7. In Theorem 6, the augmented vector $\xi(t)$ has integrating terms of activation function $f(x(t))$ which are $\int_{t-\rho_{1} r}^{t} f^{T}(x(s)) d s, \ldots, \int_{t-r}^{t-\rho_{l-1} r} f^{T}(x(s)) d s$ and $\int_{-r}^{0} \int_{t+\theta}^{t} f^{T}(x(s)) d s d \theta$. By these terms, more past history of $f(x(t))$ can be used, which lead to less conservative results.

Remark 8. Compared with those in previous articles, Ours constructed a new type of Lyapunov-Krasovskii functional which has three differences: (1) an independent augmented variable $\int_{t-h}^{t} x^{T}(s) d s$; (2) the cross terms between entries in $\xi_{1}(t), \xi_{2}(t, s)$, respectively; (3) quadratic terms multiplied by first, second, and third degrees of a scalar function $h-t+s$ by 1 means the number increase of the integral by 1 .

Remark 9. Compared with traditional approach to deal with term like $\int_{t-h}^{t} \dot{x}^{T}(s) S_{5} \dot{x}(s) d s$, Lemma 5 provides a new handling method. This new handling method can establish the relationship among $\int_{t-h(t)}^{t} x^{T}(s) d s, \int_{t-h}^{t-h(t)} x^{T}(s) d s$ and $x(t-h)$, which may significantly reduce the conservatism of stability criteria. 
Remark 10. When $W_{3}=0$, the system (5) reduces to

$$
\begin{aligned}
\dot{x}(t)= & -W_{0} x(t)+W_{1} f(x(t)) \\
& +W_{2} f(x(t-h(t)))+W_{4} \dot{x}(t-h(t)) .
\end{aligned}
$$

Similarly, based on Theorem 6, we can obtain the asymptotical stability for system (47) as follows.

Theorem 11. For given scalars $h>0$ and $h_{D}<1$, the system (47) with the neuron activation function $f(x(t))$ satisfying the condition (6) is asymptotically stable if there exists $P>0$, $R_{i}^{T}=R_{i}>0(i=1,2,3), Q_{i}^{T}=Q_{i}>0(i=1,2)$, $S_{i}^{T}=S_{i}>0(i=1, \ldots, 6)$, diagonal matrices $G_{i}=$ $\operatorname{diag}\left\{g_{i 1}, g_{i 2}, \ldots, g_{i n}\right\}>0, K_{i}=\operatorname{diag}\left\{k_{i 1}, k_{i 2}, \ldots, k_{i n}\right\}>0$, $L_{i}=\operatorname{diag}\left\{l_{i 1}, l_{i 2}, \ldots, l_{i n}\right\}>0,(i=1,2,3)$, and $M=$ $\operatorname{diag}\left\{m_{i 1}, m_{i 2}, \ldots, m_{i n}\right\}>0,(i=1,2,3,4), T_{i}(i=1, \ldots, 5)$ and $F_{i}(i=1, \ldots, 6)$ with appropriate dimensions such that the following symmetric linear matrix inequality holds:

$$
\begin{gathered}
\widehat{\Xi}_{1}=\left[\begin{array}{cccc}
\widehat{\Pi}_{0} & h F_{1}^{T} & h F_{2}^{T} & h F_{3}^{T} \\
* & -h R_{3} & 0 & 0 \\
* & * & -Q_{1} & 0 \\
* & * & * & -\frac{h}{3} Q_{2}
\end{array}\right]<0, \\
\widehat{\Xi}_{2}=\left[\begin{array}{cccc}
\widehat{\Pi}_{0}+h \widehat{\Pi}_{1} & h F_{4}^{T} & h F_{5}^{T} & h F_{6}^{T} \\
* & -h R_{3} & 0 & 0 \\
* & * & -Q_{1} & 0 \\
* & * & * & -\frac{h}{3} Q_{2}
\end{array}\right]<0,
\end{gathered}
$$

where

$$
\begin{aligned}
& \widehat{\Pi}_{0}=\left\langle 2\left[\begin{array}{ll}
\widehat{e}_{1} & \widehat{e}_{7}+\widehat{e}_{8}
\end{array}\right] P\left[\begin{array}{ll}
e_{2} & \widehat{e}_{1}-e_{4}
\end{array}\right]^{T}\right. \\
& +2\left[\begin{array}{ll}
\widehat{e}_{2} & 0
\end{array}\right] R_{1}\left[\begin{array}{ll}
0 & \widehat{e}_{8}
\end{array}\right]^{T} \\
& \left.+2\left[\begin{array}{ll}
\widehat{e}_{2} & 0
\end{array}\right] R_{2}\left[\begin{array}{ll}
h \widehat{e}_{1} & \widehat{e}_{7}+\widehat{e}_{8}
\end{array}\right]^{T}\right\rangle_{s}+\left[\begin{array}{ll}
\widehat{e}_{1} & \widehat{e}_{1}
\end{array}\right] \\
& \times\left(R_{1}+R_{2}\right)\left[\begin{array}{ll}
\widehat{e}_{1} & \widehat{e}_{1}
\end{array}\right]^{T}-\left(1-h_{D}\right)\left[\begin{array}{ll}
\widehat{e}_{1} & \widehat{e}_{3}
\end{array}\right] \\
& \times R_{1}\left[\begin{array}{ll}
\widehat{e}_{1} & \widehat{e}_{3}
\end{array}\right]^{T}-\left[\begin{array}{ll}
\widehat{e}_{1} & \hat{e}_{4}
\end{array}\right] R_{2}\left[\begin{array}{ll}
\widehat{e}_{1} & \widehat{e}_{4}
\end{array}\right]^{T}+h\left[\begin{array}{ll}
\widehat{e}_{1} & \widehat{e}_{2}
\end{array}\right] R_{3} \\
& \times\left[\begin{array}{ll}
\widehat{e}_{1} & \widehat{e}_{2}
\end{array}\right]^{T}+\widehat{e}_{2}\left(h^{2} Q_{1}+h^{3} Q_{2}\right) e_{2}^{T} \\
& +\left\langle 2 F_{1}^{T}\left[\begin{array}{ll}
\widehat{e}_{7} & \widehat{e}_{3}-\widehat{e}_{4}
\end{array}\right]^{T}+4 F_{2}^{T}\left[h \widehat{e}_{3}-\widehat{e}_{7}\right]^{T}\right. \\
& +6 F_{3}^{T}\left[h \widehat{e}_{3}-\widehat{e}_{7}\right]^{T}+2 F_{4}^{T}\left[\begin{array}{ll}
\widehat{e}_{8} & \widehat{e}_{1}-\widehat{e}_{3}
\end{array}\right]^{T}-4 F_{5}^{T} \widehat{e}_{8}^{T} \\
& \left.-6 F_{6}^{T} \widehat{e}_{8}^{T}\right\rangle_{s}+\widehat{e}_{9}\left(S_{1}+S_{2}\right) \hat{e}_{9}^{T}-\left(1-h_{D}\right) \hat{e}_{10} S_{1} \hat{e}_{10}^{T} \\
& -\widehat{e}_{11} S_{2} \widehat{e}_{11}^{T}-\widehat{e}_{6} S_{4} \widehat{e}_{6}^{T}+\widehat{e}_{2}\left(S_{3}+S_{4}\right) \hat{e}_{2}^{T} \\
& -\left(1-h_{D}\right) \widehat{e}_{5} S_{3} e_{5}^{T}+\widehat{e}_{2}\left(h S_{5}+\frac{h^{2}}{2} S_{6}\right) \hat{e}_{2}^{T}
\end{aligned}
$$

$$
\begin{aligned}
& -2 \widehat{e}_{1} S_{6} \hat{e}_{1}^{T}+\frac{4}{h} \widehat{e}_{1} S_{6} \hat{e}_{7}^{T}+\frac{4}{h^{2}} \widehat{e}_{4} S_{5} \widehat{e}_{7}^{T}+\frac{4}{h^{2}} \widehat{e}_{4} S_{5} \widehat{e}_{8}^{T} \\
& -\widehat{e}_{7}\left(\frac{2}{h^{3}} S_{5}+\frac{2}{h^{2}} S_{6}\right) \hat{e}_{7}^{T}-\widehat{e}_{7}\left(\frac{4}{h^{3}} S_{5}+\frac{4}{h^{2}} S_{6}\right) \hat{e}_{8}^{T} \\
& -\widehat{e}_{8}\left(\frac{2}{h^{3}} S_{5}+\frac{2}{h^{2}} S_{6}\right) \widehat{e}_{8}^{T}+\widehat{e}_{1}\left(\bar{\Sigma} K_{1}-\underline{\Sigma} G_{1}+L_{1} \Sigma\right) \widehat{e}_{2}^{T} \\
& +2 \widehat{e}_{2}\left(G_{1}-K_{1}+L_{1}\right) \hat{e}_{9}^{T}+2 \widehat{e}_{3}\left(\bar{\Sigma} K_{2}-\underline{\Sigma} G_{2}+L_{2} \Sigma\right) \\
& \times \widehat{e}_{5}^{T}+2 \widehat{e}_{5}\left(G_{2}-K_{2}+L_{2}\right) \hat{e}_{10}^{T} \\
& +2 \widehat{e}_{4}\left(\bar{\Sigma} K_{3}-\underline{\Sigma} G_{3}+L_{3} \Sigma\right) \hat{e}_{6}^{T}+2 \widehat{e}_{6}\left(G_{3}-K_{3}+L_{3}\right) \\
& \times \widehat{e}_{11}^{T}+e_{1}\left(\Sigma M_{1} \Sigma-M_{2} \Sigma_{1}\right) \hat{e}_{1}^{T}+2 \widehat{e}_{1} M_{2} \Sigma_{2} \widehat{e}_{9}^{T} \\
& -\widehat{e}_{3} M_{3} \Sigma_{1} \hat{e}_{3}^{T}+2 \widehat{e}_{3} M_{3} \Sigma_{2} \hat{e}_{10}^{T}-\widehat{e}_{9}\left(M_{1}+M_{2}\right) \hat{e}_{9}^{T} \\
& -\widehat{e}_{10} M_{3} \widehat{e}_{10}^{T}-\widehat{e}_{4} M_{4} \Sigma_{1} \hat{e}_{4}^{T}+2 \widehat{e}_{4} M_{4} \Sigma_{2} \widehat{e}_{11}^{T} \\
& +\widehat{e}_{2}\left(-T_{1}-T_{1}^{T}\right) \widehat{e}_{2}^{T}-\widehat{e}_{11} M_{4} \widehat{e}_{11}^{T}-2 \widehat{e}_{1} W_{0}^{T} T_{1}^{T} \widehat{e}_{2}^{T} \\
& +2 \widehat{e}_{2} T_{1} W_{1} \widehat{e}_{9}^{T}+2 \widehat{e}_{2} T_{1} W_{2} \widehat{e}_{10}^{T}-2 e_{1} T_{2} \widehat{e}_{2}^{T} \\
& +\widehat{e}_{1}\left(-T_{2} W_{0}-W_{0}^{T} T_{2}^{T}\right) \widehat{e}_{1}^{T}+2 \widehat{e}_{1} T_{2} W_{1} \widehat{e}_{9}^{T} \\
& +2 \widehat{e}_{1} T_{2} W_{2} \widehat{e}_{10}^{T}+2 \widehat{e}_{2} T_{2} W_{4} \widehat{e}_{5}^{T}-2 \widehat{e}_{1} W_{0}^{T} T_{3}^{T} \widehat{e}_{9}^{T} \\
& +2 \widehat{e}_{9} T_{3} W_{2} \widehat{e}_{10}^{T}+\widehat{e}_{9}\left(T_{3} W_{1}+W_{1}^{T} T_{3}^{T}\right) \widehat{e}_{9}^{T} \\
& +2 \widehat{e}_{5} W_{4}^{T} T_{3}^{T} \widehat{e}_{9}^{T}-2 \widehat{e}_{2} T_{4}^{T} \widehat{e}_{10}^{T}-2 \widehat{e}_{1} W_{0}^{T} T_{4}^{T} \widehat{e}_{10}^{T} \\
& +\widehat{e}_{9} W_{1}^{T} T_{4}^{T} \hat{e}_{10}^{T}+\widehat{e}_{10}\left(T_{4} W_{2}+W_{2}^{T} T_{4}^{T}\right) \hat{e}_{10}^{T} \\
& +2 \widehat{e}_{5} W_{4}^{T} T_{3}^{T} \widehat{e}_{9}^{T}-2 \widehat{e}_{2} T_{5}^{T} \widehat{e}_{5}^{T}-2 \widehat{e}_{1} W_{0}^{T} T_{5}^{T} \widehat{e}_{5}^{T} \\
& +\widehat{e}_{5} T_{5} W_{1} \widehat{e}_{9}^{T}+\widehat{e}_{5} T_{5} W_{2} \widehat{e}_{10}^{T}-\frac{2}{h} \widehat{e}_{4} S_{5} \widehat{e}_{4}^{T} \\
& +\frac{4}{h} \widehat{e}_{1} S_{6} \widehat{e}_{8}^{T}+2 \widehat{e}_{5}\left(T_{5} W_{4}+W_{4}^{T} T_{4}^{T}\right) \widehat{e}_{5}^{T} \\
& +2 \widehat{e}_{5} W_{4}^{T} T_{4}^{T} \widehat{e}_{10}^{T}+2 \widehat{e}_{2} T_{1} W_{4} \widehat{e}_{5}^{T}-2 \widehat{e}_{2} T_{3}^{T} \widehat{e}_{9}^{T}, \\
& \widehat{\Pi}_{1}=\left\langle 2\left(\begin{array}{ll}
\widehat{e}_{2} & 0
\end{array}\right) R_{1}\left(\begin{array}{ll}
\widehat{e}_{1} & 0
\end{array}\right)^{T}-4 F_{2}^{T} \widehat{e}_{3}^{T}\right. \\
& \left.-6 F_{3}^{T} \widehat{e}_{3}^{T}+4 F_{5}^{T} \widehat{e}_{1}^{T}+6 F_{6}^{T} \widehat{e}_{1}^{T}\right\rangle_{s},
\end{aligned}
$$

with

$$
\begin{gathered}
\langle\cdot\rangle_{s}=\frac{1}{2}\left[(\cdot)+(\cdot)^{T}\right], \\
\widehat{e}_{i}=\left[\begin{array}{lll}
0_{n \times(i-1)} & I_{n \times n} & 0_{n \times(11)}
\end{array}\right]^{T} \quad(i=1, \ldots, 11) .
\end{gathered}
$$

Remark 12. When $W_{4}=0$, the system (5) reduces to

$$
\begin{aligned}
\dot{x}(t)= & -W_{0} x(t)+W_{1} f(x(t)) \\
& +W_{2} f(x(t-h(t)))+W_{3} \int_{t-r}^{t} f^{T}(x(s)) d s .
\end{aligned}
$$


Similarly, based on Theorem 6, we can obtain the asymptotical stability for system (52) as follows.

Theorem 13. For given scalars $h>0$ and $h_{D}<1$, the system (52) with the neuron activation function $f(x(t))$ satisfying the condition (6) is asymptotically stable if there exists $P>0$, $R_{i}^{T}=R_{i}>0(i=1,2,3), Q_{i}^{T}=Q_{i}>0(i=1,2), S_{i}^{T}=$ $S_{i}>0(i=1,2,5 \ldots, 8), Z_{i}^{T}=Z_{i}>0(i=1, \ldots, l)$, diagonal matrices $G_{1}=\operatorname{diag}\left\{g_{1}, g_{2}, \ldots, g_{n}\right\}>0, K_{1}=$ $\operatorname{diag}\left\{k_{1}, k_{2}, \ldots, k_{n}\right\}>0, L_{1}=\operatorname{diag}\left\{l_{1}, l_{2}, \ldots, l_{n}\right\}>0$, and $M=$ $\operatorname{diag}\left\{m_{i 1}, m_{i 2}, \ldots, m_{i n}\right\}>0,(i=1,2,3,4), T_{i}(i=1, \ldots, 4)$ and $F_{i}(i=1, \ldots, 6)$ with appropriate dimensions such that the following symmetric linear matrix inequality holds:

$$
\begin{gathered}
\widetilde{\Xi}_{1}=\left[\begin{array}{cccc}
\widetilde{\Pi}_{0} & h F_{1}^{T} & h F_{2}^{T} & h F_{3}^{T} \\
* & -h R_{3} & 0 & 0 \\
* & * & -Q_{1} & 0 \\
* & * & * & -\frac{h}{3} Q_{2}
\end{array}\right]<0, \\
\widetilde{\Xi}_{2}=\left[\begin{array}{cccc}
\widetilde{\Pi}_{0}+h \widetilde{\Pi}_{1} & h F_{4}^{T} & h F_{5}^{T} & h F_{6}^{T} \\
* & -h R_{3} & 0 & 0 \\
* & * & -Q_{1} & 0 \\
* & * & * & -\frac{h}{3} Q_{2}
\end{array}\right]<0,
\end{gathered}
$$

where

$$
\begin{aligned}
& \widetilde{\Pi}_{0}=\left\langle 2\left[\begin{array}{ll}
\widetilde{e}_{1} & \widetilde{e}_{5}+\widetilde{e}_{6}
\end{array}\right] P\left[\begin{array}{cc}
\widetilde{e}_{2} & \widetilde{e}_{1}-\widetilde{e}_{4}
\end{array}\right]^{T}\right. \\
& +2\left[\begin{array}{ll}
\widetilde{e}_{2} & 0
\end{array}\right] R_{1}\left[\begin{array}{ll}
0 & \widetilde{e}_{6}
\end{array}\right]^{T}+2\left[\begin{array}{ll}
\widetilde{e}_{2} & 0
\end{array}\right] \\
& \left.\times R_{2}\left[\begin{array}{ll}
h \widetilde{e}_{1} & \widetilde{e}_{5}+\widetilde{e}_{6}
\end{array}\right]^{T}\right\rangle_{s}+\left[\begin{array}{ll}
\widetilde{e}_{1} & \widetilde{e}_{1}
\end{array}\right] \\
& \times\left(R_{1}+R_{2}\right)\left[\begin{array}{ll}
\widetilde{e}_{1} & \widetilde{e}_{1}
\end{array}\right]^{T}-\left(1-h_{D}\right)\left[\begin{array}{ll}
\widetilde{e}_{1} & e_{3}
\end{array}\right] \\
& \times R_{1}\left[\begin{array}{ll}
\widetilde{e}_{1} & \widetilde{e}_{3}
\end{array}\right]^{T}-\left[\begin{array}{ll}
\widetilde{e}_{1} & e_{4}
\end{array}\right] R_{2}\left[\begin{array}{ll}
\widetilde{e}_{1} & \widetilde{e}_{4}
\end{array}\right]^{T} \\
& +h\left[\begin{array}{ll}
\widetilde{e}_{1} & \widetilde{e}_{2}
\end{array}\right] R_{3} \times\left[\begin{array}{ll}
\widetilde{e}_{1} & \widetilde{e}_{2}
\end{array}\right]^{T}+\widetilde{e}_{2}\left(h^{2} Q_{1}+h^{3} Q_{2}\right) \\
& \times \widetilde{e}_{2}^{T}+\left\langle 2 F_{1}^{T}\left[\widetilde{e}_{5} \widetilde{e}_{3}-\widetilde{e}_{4}\right]^{T}+4 F_{2}^{T}\left[h \widetilde{e}_{3}-\widetilde{e}_{5}\right]^{T}\right. \\
& +6 F_{3}^{T}\left[h \widetilde{e}_{3}-\widetilde{e}_{5}\right]^{T}+2 F_{4}^{T}\left[\begin{array}{ll}
\tilde{e}_{6} & \widetilde{e}_{1}-\widetilde{e}_{3}
\end{array}\right]^{T} \\
& \left.-4 F_{5}^{T} \widetilde{e}_{6}^{T}-6 F_{6}^{T} \widetilde{e}_{6}^{T}\right\rangle_{s}+\widetilde{e}_{7}\left(S_{1}+S_{2}\right) \widetilde{e}_{7}^{T} \\
& -\left(1-h_{D}\right) \widetilde{e}_{8} S_{1} \widetilde{e}_{8}^{T}-\widetilde{e}_{9} S_{2} \widetilde{e}_{9}^{T}+\widetilde{e}_{2}\left(h S_{5}+\frac{h^{2}}{2} S_{6}\right) \\
& \times \widetilde{e}_{2}^{T}-2 \widetilde{e}_{1} S_{6} \widetilde{e}_{1}^{T}+\frac{4}{h} \widetilde{e}_{1} S_{6} \widetilde{e}_{5}^{T}+\widetilde{e}_{7} W_{1}^{T} T_{4}^{T} \widetilde{e}_{8}^{T} \\
& +\widetilde{e}_{8}\left(T_{4} W_{2}+W_{2}^{T} T_{4}^{T}\right) \widetilde{e}_{8}^{T}+\frac{4}{h} \widetilde{e}_{1} \times S_{6} \widetilde{e}_{6}^{T}+\frac{4}{h^{2}} \widetilde{e}_{4} S_{5} \widetilde{e}_{5}^{T} \\
& +\frac{4}{h^{2}} \widetilde{e}_{4} S_{5} \widetilde{e}_{6}^{T}-\frac{2}{h} \widetilde{e}_{4} S_{5} e_{4}^{T}-\widetilde{e}_{5}\left(\frac{2}{h^{3}} S_{5}+\frac{2}{h^{2}} S_{6}\right) \widetilde{e}_{5}^{T} \\
& -\widetilde{e}_{5}\left(\frac{4}{h^{3}} S_{5}+\frac{4}{h^{2}} S_{6}\right) \tilde{e}_{6}^{T}-\widetilde{e}_{6}\left(\frac{2}{h^{3}} S_{5}+\frac{2}{h^{2}} S_{6}\right) \tilde{e}_{6}^{T}
\end{aligned}
$$

$$
\begin{aligned}
& +\widetilde{e}_{7}\left(r S_{7}+\frac{r^{2}}{2} S_{8}+\sum_{i=1}^{l}\left(\rho_{i}-\rho_{i-1}\right) r Z_{i}\right) \widetilde{e}_{7}^{T} \\
& -\sum_{i=1}^{l} \frac{1}{\left(\rho_{i}-\rho_{i-1}\right) r} \widetilde{e}_{9+i} Z_{i} \tilde{e}_{9+i}^{T}-\frac{1}{r}\left(\widetilde{e}_{10}+\cdots+\widetilde{e}_{9+l}\right) \\
& \times S_{7}\left(\widetilde{e}_{10}+\cdots+e_{9+l}\right)^{T}-\frac{2}{r^{2}} \widetilde{e}_{10+l} S_{8} \widetilde{e}_{10+l}^{T} \\
& +\widetilde{e}_{1}\left(\bar{\Sigma} K_{1}-\underline{\Sigma} G_{1}+L_{1} \Sigma\right) \tilde{e}_{2}^{T}+2 \widetilde{e}_{2}\left(G_{1}-K_{1}+L_{1}\right) e_{7}^{T} \\
& +\widetilde{e}_{1}\left(\Sigma M_{1} \Sigma-M_{2} \Sigma_{1}\right) e_{1}^{T}+2 e_{1} M_{2} \Sigma_{2} e_{7}^{T}-e_{3} M_{3} \Sigma_{1} e_{3}^{T} \\
& +2 e_{7} T_{3} W_{2} e_{8}^{T}+2 \widetilde{e}_{3} M_{3} \Sigma_{2} \widetilde{e}_{8}^{T}-\widetilde{e}_{7}\left(M_{1}+M_{2}\right) \tilde{e}_{7}^{T} \\
& -\widetilde{e}_{8} M_{3} \widetilde{e}_{8}^{T}-\widetilde{e}_{4} M_{4} \Sigma_{1} \widetilde{e}_{4}^{T}+2 \widetilde{e}_{4} M_{4} \Sigma_{2} \widetilde{e}_{9}^{T} \\
& +\widetilde{e}_{2}\left(-T_{1}-T_{1}^{T}\right) \widetilde{e}_{2}^{T}-\widetilde{e}_{9} M_{4} \tilde{e}_{9}^{T}-2 \widetilde{e}_{1} W_{0}^{T} T_{1}^{T} \widetilde{e}_{2}^{T} \\
& +2 e_{2} T_{1} W_{1} \tilde{e}_{7}^{T}+2 \widetilde{e}_{2} T_{1} W_{2} \tilde{e}_{8}^{T}+2 \widetilde{e}_{2} T_{1} W_{3} \\
& \times\left(\widetilde{e}_{10}+\cdots+\widetilde{e}_{9+l}\right)^{T}-2 \widetilde{e}_{1} \times W_{0}^{T} T_{4}^{T} \widetilde{e}_{8}^{T}-2 \widetilde{e}_{1} T_{2} e_{2}^{T} \\
& +\widetilde{e}_{1}\left(-T_{2} W_{0}-W_{0}^{T} T_{2}^{T}\right) \widetilde{e}_{1}^{T}+2 \widetilde{e}_{1} T_{2} W_{1} \widetilde{e}_{7}^{T} \\
& +2 \widetilde{e}_{1} T_{2} W_{2} e_{8}^{T}-2 \widetilde{e}_{2} T_{4}^{T} \widetilde{e}_{8}^{T}+2 \widetilde{e}_{1} T_{2} W_{3} \\
& \times\left(\widetilde{e}_{10}+\cdots+\widetilde{e}_{9+l}\right)^{T}-2 \widetilde{e}_{2} T_{3}^{T} \widetilde{e}_{7}^{T}-2 e_{1} W_{0}^{T} T_{3}^{T} \widetilde{e}_{7}^{T} \\
& +\widetilde{e}_{7}\left(T_{3} W_{1}+W_{1}^{T} T_{3}^{T}\right) \tilde{e}_{7}^{T} \\
& +2 \widetilde{e}_{7} T_{3} W_{3}\left(\widetilde{e}_{10}+\cdots+e_{9+l}\right)^{T} \\
& +2 \widetilde{e}_{8} T_{4} W_{3}\left(\widetilde{e}_{10}+\cdots+\widetilde{e}_{9+l}\right)^{T}, \\
& \widetilde{\Pi}_{1}=\left\langle 2\left(\begin{array}{ll}
\widetilde{e}_{2} & 0
\end{array}\right) R_{1}\left(\begin{array}{ll}
\widetilde{e}_{1} & \widetilde{e}_{0}
\end{array}\right)^{T}-4 F_{2}^{T} \widetilde{e}_{3}^{T}\right. \\
& \left.-6 F_{3}^{T} \widetilde{e}_{3}^{T}+4 F_{5}^{T} \widetilde{e}_{1}^{T}+6 F_{6}^{T} \widetilde{e}_{1}^{T}\right\rangle_{s},
\end{aligned}
$$

with

$$
\begin{gathered}
\langle\cdot\rangle_{s}=\frac{1}{2}\left[(\cdot)+(\cdot)^{T}\right], \\
\widetilde{e}_{i}=\left[\begin{array}{lll}
0_{n \times(i-1)} & I_{n \times n} & 0_{n \times(10+l-i)}
\end{array}\right]^{T} \quad(i=1, \ldots, 10+l) .
\end{gathered}
$$

Remark 14. When $W_{3}=0$ and $W_{4}=0$, the system (5) reduces to

$$
\dot{x}(t)=-W_{0} x(t)+W_{1} f(x(t))+W_{2} f(x(t-h(t))) .
$$

Similarly, based on Theorem 6, we can obtain the asymptotical stability for system (57) as follows.

Theorem 15. For given scalars $h>0$ and $h_{D}<1$, the system (57) with the neuron activation function $f(x(t))$ satisfying the condition (6) is asymptotically stable if there exists $P>0, R_{i}^{T}=$ $R_{i}>0(i=1,2,3), Q_{i}^{T}=Q_{i}>0(i=1,2), S_{i}^{T}=S_{i}>0(i=$ $1,2,5,6)$, diagonal matrices $G_{1}=\operatorname{diag}\left\{g_{i 1}, g_{2}, \ldots, g_{i n}\right\}>0$, 
$K_{1}=\operatorname{diag}\left\{k_{1}, k_{2}, \ldots, k_{n}\right\}>0, L_{1}=\operatorname{diag}\left\{l_{1}, l_{2}, \ldots, l_{n}\right\}>0$, and $M=\operatorname{diag}\left\{m_{i 1}, m_{i 2}, \ldots, m_{i n}\right\}>0,(i=1,2,3,4), T_{i}(i=$ $1, \ldots, 4)$ and $F_{i}(i=1, \ldots, 6)$ with appropriate dimensions such that the following symmetric linear matrix inequality holds:

$$
\begin{gathered}
\breve{\Xi}_{1}=\left[\begin{array}{cccc}
\breve{\Pi}_{0} & h F_{1}^{T} & h F_{2}^{T} & h F_{3}^{T} \\
* & -h R_{3} & 0 & 0 \\
* & * & -Q_{1} & 0 \\
* & * & * & -\frac{h}{3} Q_{2}
\end{array}\right]<0, \\
\breve{\Xi}_{2}=\left[\begin{array}{cccc}
\breve{\Pi}_{0}+h \breve{\Pi}_{1} & h F_{4}^{T} & h F_{5}^{T} & h F_{6}^{T} \\
* & -h R_{3} & 0 & 0 \\
* & * & -Q_{1} & 0 \\
* & * & * & -\frac{h}{3} Q_{2}
\end{array}\right]<0,
\end{gathered}
$$

where

$$
\begin{aligned}
& \breve{\Pi}_{0}=\left\langle 2\left[\begin{array}{ll}
\breve{e}_{1} & \breve{e}_{5}+\breve{e}_{6}
\end{array}\right] P\left[\begin{array}{ll}
e_{2} & \breve{e}_{1}-e_{4}
\end{array}\right]^{T}+2\left[\begin{array}{ll}
\breve{e}_{2} & 0
\end{array}\right] R_{1}\left[\begin{array}{ll}
0 & e_{6}
\end{array}\right]^{T}\right. \\
& \left.+2\left[\begin{array}{ll}
\breve{e}_{2} & 0
\end{array}\right] R_{2}\left[\begin{array}{ll}
h \breve{e}_{1} & \breve{e}_{5}+\breve{e}_{6}
\end{array}\right]^{T}\right\rangle_{s}+\left[\begin{array}{ll}
\breve{e}_{1} & \widehat{e}_{1}
\end{array}\right] \\
& \times\left(R_{1}+R_{2}\right)\left[\begin{array}{ll}
\breve{e}_{1} & \breve{e}_{1}
\end{array}\right]^{T}-\left(1-h_{D}\right)\left[\begin{array}{ll}
\breve{e}_{1} & \breve{e}_{3}
\end{array}\right] R_{1}\left[\begin{array}{ll}
\breve{e}_{1} & \breve{e}_{3}
\end{array}\right]^{T} \\
& \text { - }\left[\begin{array}{ll}
\breve{e}_{1} & \breve{e}_{4}
\end{array}\right] R_{2}\left[\begin{array}{ll}
\breve{e}_{1} & \breve{e}_{4}
\end{array}\right]^{T}+h\left[\begin{array}{ll}
\breve{e}_{1} & \breve{e}_{2}
\end{array}\right] R_{3} \\
& \times\left[\begin{array}{ll}
\breve{e}_{1} & \breve{e}_{2}
\end{array}\right]^{T}+\breve{e}_{2}\left(h^{2} Q_{1}+h^{3} Q_{2}\right) e_{2}^{T} \\
& +\left\langle 2 F_{1}^{T}\left[\breve{e}_{5} \breve{e}_{3}-\breve{e}_{4}\right]^{T}+4 F_{2}^{T}\left[h \breve{e}_{3}-\breve{e}_{5}\right]^{T}\right. \\
& +6 F_{3}^{T}\left[h \breve{e}_{3}-\breve{e}_{5}\right]^{T}+2 F_{4}^{T}\left[\breve{e}_{6} \breve{e}_{1}-\breve{e}_{3}\right]^{T} \\
& \left.-4 F_{5}^{T} \breve{e}_{6}^{T}-6 F_{6}^{T} \breve{e}_{6}^{T}\right\rangle_{s}+\breve{e}_{7}\left(S_{1}+S_{2}\right) \breve{e}_{7}^{T} \\
& -\left(1-h_{D}\right) \breve{e}_{8} S_{1} \breve{e}_{8}^{T}-\breve{e}_{9} S_{2} \breve{e}_{9}^{T}+\breve{e}_{2}\left(h S_{5}+\frac{h^{2}}{2} S_{6}\right) \breve{e}_{2}^{T} \\
& -2 \breve{e}_{1} S_{6} \breve{e}_{1}^{T}+\frac{2}{h} \breve{e}_{1} S_{6} \breve{e}_{5}^{T}+\frac{2}{h} \breve{e}_{1} S_{6} \breve{e}_{6}^{T}+\frac{2}{h^{2}} \breve{e}_{4} S_{5} \breve{e}_{5}^{T} \\
& +\frac{2}{h^{2}} \breve{e}_{4} S_{5} \breve{e}_{6}^{T}-\frac{2}{h} \breve{e}_{4} S_{5} \breve{e}_{4}^{T}-\breve{e}_{5}\left(\frac{2}{h^{3}} S_{5}+\frac{2}{h^{2}} S_{6}\right) \breve{e}_{5}^{T} \\
& -\breve{e}_{5}\left(\frac{2}{h^{3}} S_{5}+\frac{2}{h^{2}} S_{6}\right) \breve{e}_{6}^{T}-\breve{e}_{6}\left(\frac{2}{h^{3}} S_{5}+\frac{2}{h^{2}} S_{6}\right) \breve{e}_{6}^{T} \\
& +2 \breve{e}_{2}\left(G_{1}-K_{1}+L_{1}\right) \breve{e}_{7}^{T}+\breve{e}_{1}\left(\bar{\Sigma} K_{1}-\underline{\Sigma} G_{1}+L_{1} \Sigma\right) \breve{e}_{2}^{T} \\
& +\breve{e}_{1}\left(\Sigma M_{1} \Sigma-M_{2} \Sigma_{1}\right) \breve{e}_{1}^{T}+2 \breve{e}_{1} M_{2} \Sigma_{2} \breve{e}_{7}^{T}-\breve{e}_{3} M_{3} \Sigma_{1} \breve{e}_{3}^{T} \\
& +2 \breve{e}_{3} M_{3} \Sigma_{2} \hat{e}_{8}^{T}-\breve{e}_{7}\left(M_{1}+M_{2}\right) \breve{e}_{7}^{T} \\
& -\breve{e}_{8} M_{3} \breve{e}_{8}^{T}-\breve{e}_{4} M_{4} \Sigma_{1} \breve{e}_{4}^{T}+2 \breve{e}_{4} M_{4} \Sigma_{2} \hat{e}_{9}^{T} \\
& +\breve{e}_{2}\left(-T_{1}-T_{1}^{T}\right) \breve{e}_{2}^{T}-\breve{e}_{9} M_{4} \breve{e}_{9}^{T}-2 \breve{e}_{1} W_{0}^{T} T_{1}^{T} \breve{e}_{2}^{T} \\
& +2 \breve{e}_{2} T_{1} W_{1} \breve{e}_{7}^{T}+2 \breve{e}_{2} T_{1} W_{2} \breve{e}_{8}^{T}-2 e_{1} T_{2} \breve{e}_{2}^{T} \\
& +\breve{e}_{1}\left(-T_{2} W_{0}-W_{0}^{T} T_{2}^{T}\right) \breve{e}_{1}^{T}+2 \breve{e}_{1} T_{2} W_{2} \breve{e}_{8}^{T}
\end{aligned}
$$

$$
\begin{aligned}
& -2 \breve{e}_{2} T_{3}^{T} \breve{e}_{7}^{T}-2 \breve{e}_{1} W_{0}^{T} T_{3}^{T} \breve{e}_{7}^{T}+2 \breve{e}_{7} T_{3} W_{2} \breve{e}_{8}^{T} \\
& +\breve{e}_{7}\left(T_{3} W_{1}+W_{1}^{T} T_{3}^{T}\right) \breve{e}_{7}^{T}-2 \breve{e}_{2} T_{4}^{T} \breve{e}_{8}^{T} \\
& -2 \breve{e}_{1} W_{0}^{T} T_{4}^{T} \breve{e}_{8}^{T}+\breve{e}_{7} W_{1}^{T} T_{4}^{T} \breve{e}_{8}^{T} \\
& +\breve{e}_{8}\left(T_{4} W_{2}+W_{2}^{T} T_{4}^{T}\right) \breve{e}_{8}^{T}+2 \breve{e}_{1} T_{2} W_{1} \breve{e}_{7}^{T}, \\
\widehat{\Pi}_{1}=\langle & \left\langle\left(\begin{array}{ll}
\widehat{e}_{2} & 0
\end{array}\right) R_{1}\left(\widehat{e}_{1} 0\right)^{T}-4 F_{2}^{T} \widehat{e}_{3}^{T}-6 F_{3}^{T} \widehat{e}_{3}^{T}\right. \\
& \left.+4 F_{5}^{T} \widehat{e}_{1}^{T}+6 F_{6}^{T} \widehat{e}_{1}^{T}\right\rangle_{s}
\end{aligned}
$$

with

$$
\langle\cdot\rangle_{s}=\frac{1}{2}\left[(\cdot)+(\cdot)^{T}\right],
$$

$$
\widehat{e}_{i}=\left[\begin{array}{lll}
0_{n \times(i-1)} & I_{n \times n} & 0_{n \times(9 i)}
\end{array}\right]^{T} \quad(i=1, \ldots, 9) .
$$

\section{Numerical Examples}

In this section, four numerical examples are given to show the effectiveness and improvement of the main results proposed in the paper.

Example 1. Consider the following neural networks of neutral type with discrete and distributed delays:

$$
\begin{aligned}
\dot{x}(t)= & -W_{0} x(t)+W_{1} f(x(t))+W_{2} f(x(t-h(t))) \\
& +W_{3} \int_{t-r}^{t} f^{T}(x(s)) d s+W_{4} \dot{x}(t-h(t)),
\end{aligned}
$$

where

$$
\begin{aligned}
& W_{1}=\left[\begin{array}{cccc}
-2.5573 & -1.3813 & 1.9574 & -1.1398 \\
-1.0226 & -0.8845 & 0.5045 & -0.2111 \\
1.0378 & 1.5532 & 0.6645 & 1.1902 \\
-0.3896 & 0.7079 & -0.3398 & -2.2543
\end{array}\right], \\
& W_{2}=\left[\begin{array}{cccc}
0.2853 & -0.0793 & 0.4694 & 0.5354 \\
-0.5955 & 1.3352 & -0.9036 & 0.5529 \\
-0.1497 & -0.6065 & -0.1641 & -0.2037 \\
-0.4348 & -1.3474 & -0.6275 & -2.2543
\end{array}\right] \text {, } \\
& W_{3}=\left[\begin{array}{cccc}
0.0265 & 0.1157 & 0.0578 & -0.0930 \\
0.3186 & -0.1363 & -0.0859 & 0.0742 \\
0.2037 & -0.2049 & 0.0112 & 0.1457 \\
-0.3161 & -0.2469 & -0.0736 & -2.2543
\end{array}\right] \text {, } \\
& W_{4}=\left[\begin{array}{cccc}
-0.3054 & 0.3682 & 0.1761 & -0.0235 \\
-0.0546 & -0.2089 & -0.0754 & 0.2668 \\
0.4563 & 0.0023 & 0.1440 & 0.6928 \\
-0.0115 & -0.2349 & 0.2004 & 0.1574
\end{array}\right] \text {, } \\
& W_{0}=\operatorname{diag}\{1.6305,1.9221,2.5973,1.3775\} \text {, } \\
& \bar{\Sigma}=\operatorname{diag}\{1.0275,0.9960,0.3223,0.2113\} \text {, } \\
& \underline{\Sigma}=\operatorname{diag}\{0,0,0,0\} .
\end{aligned}
$$


TABLE 1: Maximum allowable time-delay bounds $h=r$ in Example 1.

\begin{tabular}{lccccc}
\hline$h_{D}=0$ & {$[32]$} & $(m=l=2)[39]$ & $(m=l=3)[39]$ & $(l=2)$ Theorem 6 & $(l=3)$ Theorem 6 \\
\hline$h=r$ & 1.8630 & 2.7442 & 3.0942 & 4.6531 & 4.8652 \\
\hline
\end{tabular}

TABLE 2: Maximum allowable time-delay bounds $h=r$ for different values $h_{D}$ in Example 1 .

\begin{tabular}{|c|c|c|c|c|c|c|c|c|c|}
\hline$h_{D}$ & 0.1 & 0.2 & 0.3 & 0.4 & 0.5 & 0.6 & 0.7 & 0.8 & 0.9 \\
\hline Theorem $6(l=2)$ & 4.3682 & 4.1238 & 3.9862 & 3.6782 & 3.5132 & 3.1230 & 2.8623 & 2.5219 & 1.6725 \\
\hline Theorem $6(l=3)$ & 4.5132 & 4.2685 & 4.1032 & 3.8658 & 3.7216 & 3.3275 & 2.9273 & 2.6312 & 1.7321 \\
\hline
\end{tabular}

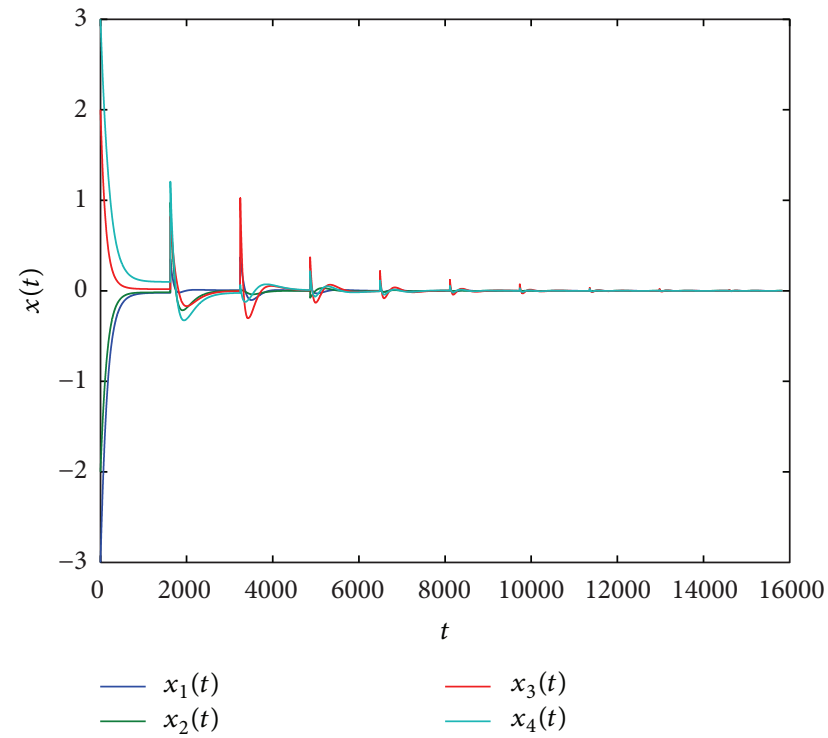

FIGURE 1: The simulation of Example 1 for $h=r=4.8652$, where the initial value is $[-3,-2,2,3]^{T}, h_{D}=0, l=3$.

From Table 1, it can be seen that the stability criterion proposed in the paper is less conservative than those obtained by Feng et al. in [32] and Lakshmanan et al. in [39]. Besides, from Tables 1 and 2, the maximum allowable time-delay bounds $h$ will become larger with the values of $l$ becoming larger. Moreover, Figures 1 and 2 show that the state vector $x(t)$ stabilizes to zero asymptotically with different $h_{D}$ and initial values. By using the Matlab LMI toolbox, we solve LMIs (13) and (14) for the case $h_{D}=0$ and $h=4.6531$ and obtain

$$
\begin{aligned}
P_{11} & =\left[\begin{array}{cccc}
0.0048 & -0.0009 & 0.0017 & -0.0003 \\
-0.0009 & 0.0072 & -0.0010 & 0.0004 \\
0.0017 & -0.0010 & 0.0045 & -0.0009 \\
-0.0003 & 0.0004 & -0.0009 & 0.0042
\end{array}\right], \\
R_{211} & =\left[\begin{array}{cccc}
0.0022 & -0.0012 & 0.0013 & -0.0004 \\
-0.0012 & 0.0043 & -0.0013 & 0.0006 \\
0.0013 & -0.0013 & 0.0021 & -0.0007 \\
-0.0004 & 0.0006 & -0.0007 & 0.0015
\end{array}\right], \\
R_{222} & =\left[\begin{array}{cccc}
0.0063 & -0.0006 & 0.0011 & -0.0007 \\
-0.0006 & 0.0085 & -0.0015 & 0.0006 \\
0.0011 & -0.0015 & 0.0050 & -0.0010 \\
-0.0007 & 0.0006 & -0.0010 & 0.0034
\end{array}\right],
\end{aligned}
$$

$$
\begin{aligned}
& R_{311}=\left[\begin{array}{cccc}
0.0030 & -0.0004 & 0.0006 & -0.0005 \\
-0.0004 & 0.0041 & -0.0009 & 0.0004 \\
0.0006 & -0.0009 & 0.0026 & -0.0006 \\
-0.0005 & 0.0004 & -0.0006 & 0.0018
\end{array}\right] \text {, } \\
& Z_{1}=\left[\begin{array}{cccc}
0.0307 & -0.0024 & -0.0035 & -0.0050 \\
-0.0024 & 0.0181 & 0.0044 & -0.0137 \\
-0.0035 & 0.0044 & 0.0136 & -0.0027 \\
-0.0050 & -0.0137 & -0.0027 & 0.0302
\end{array}\right] \text {, } \\
& Z_{2}=\left[\begin{array}{cccc}
0.0022 & -0.0000 & -0.0003 & 0.0005 \\
-0.0000 & 0.0017 & -0.0000 & -0.0001 \\
-0.0003 & -0.0000 & 0.0026 & 0.0010 \\
0.0005 & -0.0001 & 0.0010 & 0.0045
\end{array}\right] \text {, } \\
& S_{1}=\left[\begin{array}{cccc}
0.0065 & 0.0033 & 0.0006 & -0.0023 \\
0.0033 & 0.0058 & 0.0017 & -0.0031 \\
0.0006 & 0.0017 & 0.0049 & -0.0010 \\
-0.0023 & -0.0031 & -0.0010 & 0.0073
\end{array}\right] \text {, } \\
& S_{2}=\left[\begin{array}{cccc}
0.0066 & -0.0003 & -0.0016 & 0.0010 \\
-0.0003 & 0.0049 & 0.0006 & -0.0003 \\
-0.0016 & 0.0006 & 0.0052 & 0.0011 \\
0.0010 & -0.0003 & 0.0011 & 0.0063
\end{array}\right] \text {, } \\
& T_{1}=\left[\begin{array}{cccc}
0.0087 & -0.0039 & 0.0032 & -0.0014 \\
-0.0037 & 0.0144 & -0.0017 & 0.0021 \\
0.0030 & -0.0016 & 0.0061 & -0.0009 \\
-0.0007 & 0.0012 & -0.0017 & 0.0092
\end{array}\right] \text {, } \\
& T_{2}=\left[\begin{array}{cccc}
0.0131 & -0.0008 & 0.0044 & -0.0002 \\
-0.0043 & 0.0237 & -0.0074 & 0.0003 \\
-0.0012 & 0.0029 & 0.0099 & -0.0003 \\
-0.0016 & 0.0014 & -0.0025 & 0.0086
\end{array}\right] .
\end{aligned}
$$

Example 2. Using this example, we will show the improvement of our results for (52) by two cases (A) and (B). Consider the following parameters as in $[32,39]$ :

Case A

$$
\begin{gathered}
W_{0}=\left[\begin{array}{lll}
6 & 0 & 0 \\
0 & 5 & 0 \\
0 & 0 & 7
\end{array}\right], \\
W_{1}=\left[\begin{array}{ccc}
1.2 & -0.8 & 0.6 \\
0.5 & -1.5 & 0.7 \\
-0.8 & -1.2 & -1.4
\end{array}\right],
\end{gathered}
$$


TABLE 3: Maximum allowable time-delay upper bounds $h=r$ for $h_{D}=0$ in Example 2 .

\begin{tabular}{lccccccc}
\hline$h_{D}=0$ & {$[9]$} & {$[45]$} & {$[46]$} & {$[12]$} & {$[21]$} & {$[39]$} & Theorem 13 \\
\hline Case A & 4.3163 & 4.4697 & 4.3324 & 4.8374 & 4.4879 & 4.3763 & 4.9532 \\
Case B & 2.8266 & 2.9137 & 2.8317 & 2.7953 & 6.8279 & 7.2368 & 7.8698 \\
\hline
\end{tabular}

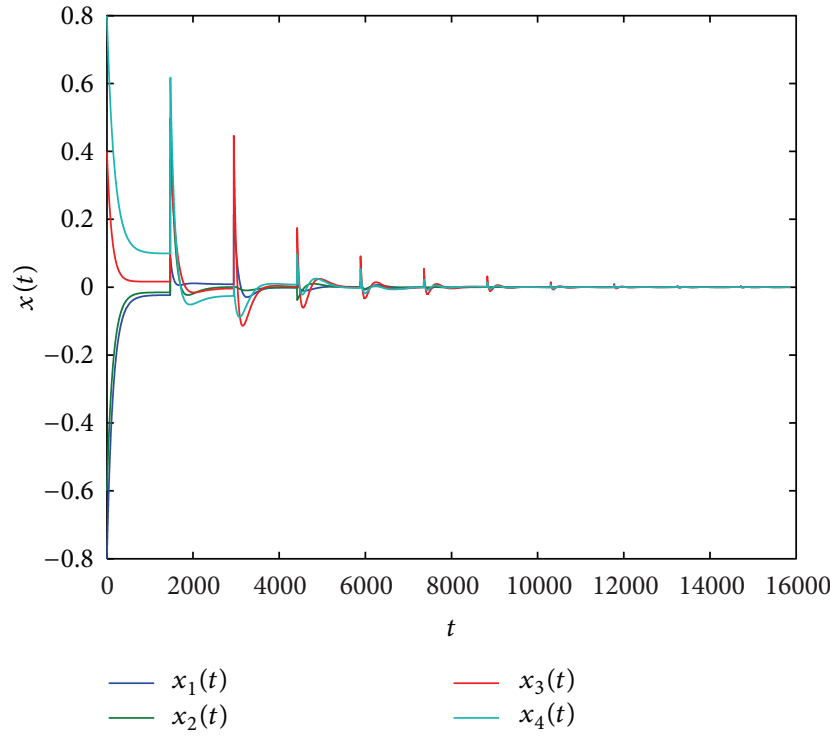

FIgURE 2: The simulation of Example 1 for $h=r=4.5312$, where the initial value is $[-0.8,-0.6,0.4,0.8]^{T}, h_{D}=0.1, l=3$.

$$
\begin{gathered}
W_{2}=\left[\begin{array}{ccc}
-1.4 & 0.9 & 0.5 \\
-0.6 & 1.2 & 0.8 \\
0.5 & -0.7 & 1.1
\end{array}\right], \\
W_{3}=\left[\begin{array}{ccc}
-1.8 & 0.7 & -0.8 \\
0.6 & 1.4 & 1 \\
-0.4 & -0.6 & 1.2
\end{array}\right], \\
\bar{\Sigma}=\left[\begin{array}{ccc}
0.5 & 0 & 0 \\
0 & 0.5 & 0 \\
0 & 0 & 0.5
\end{array}\right], \quad \underline{\Sigma}=\left[\begin{array}{lll}
0 & 0 & 0 \\
0 & 0 & 0 \\
0 & 0 & 0
\end{array}\right] .
\end{gathered}
$$

Case B

$$
\begin{array}{cc}
W_{0}=\left[\begin{array}{cc}
0.9 & 0 \\
0 & 0.8
\end{array}\right], & W_{1}=\left[\begin{array}{cc}
1 & -1.7 \\
-1.6 & 1
\end{array}\right], \\
W_{2}=\left[\begin{array}{cc}
1 & 0.6 \\
0.5 & 0.8
\end{array}\right], \quad W_{3}=\left[\begin{array}{ll}
0.4 & 0.3 \\
0.1 & 0.2
\end{array}\right], \\
\bar{\Sigma}=\left[\begin{array}{cc}
0.25 & 0 \\
0 & 0.25
\end{array}\right], \quad \underline{\Sigma}=\left[\begin{array}{ll}
0 & 0 \\
0 & 0
\end{array}\right] .
\end{array}
$$

For the above two Cases A and B of numerical examples, the maximum allowable upper bounds for guaranteeing the asymptotical stability of the corresponding systems obtained from Theorem 13 are listed in Table 3. Table 3 clearly shows

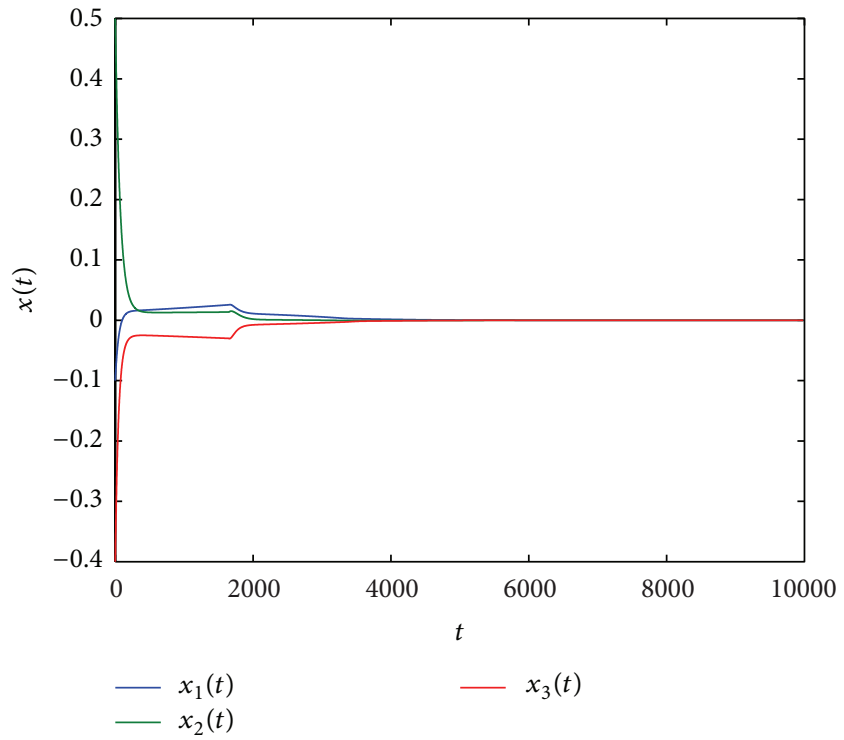

FIgURE 3: The simulation of Example 2 of Case A for $h=r=4.9532$, where the initial value is $[-0.2,0.5,-0.4]^{T}, h_{D}=0$.

that our results have larger improvement over those results of previous literature. In Case $\mathrm{A}$, when $h_{D}=0, h=r$, the simulation result is given as Figure 3, which implies that under the given conditions, the state vector $x(t)$ stabilizes to zero asymptotically.

Example 3. Consider the following delayed neural network given in (47) with parameters:

$$
\begin{array}{cc}
W_{0}=\left[\begin{array}{ll}
2 & 0 \\
0 & 2
\end{array}\right], & W_{1}=\left[\begin{array}{ll}
1.6 & 0.3 \\
0.3 & 0.5
\end{array}\right], \\
W_{2}=\left[\begin{array}{ll}
0.2 & 0.1 \\
0.1 & 0.2
\end{array}\right], & W_{4}=\left[\begin{array}{cc}
0.15 & 0 \\
0 & 0.15
\end{array}\right], \\
\bar{\Sigma}=\left[\begin{array}{ll}
1 & 0 \\
0 & 1
\end{array}\right], & \underline{\Sigma}=\left[\begin{array}{ll}
0 & 0 \\
0 & 0
\end{array}\right] .
\end{array}
$$

The corresponding results for maximum allowable upper bounds of the time-varying delay $h(t)$ are given in Table 4 . From Table 4, it can be clearly seen that the maximum allowable time-delay bounds $h$ will become small with the values of $h_{D}$ becoming large. Furthermore, when $h_{D}=0.1$, the state trajectories of the system (47) are shown in Figure 4.

Example 4. In this Example, we give four Cases (A)-(C) that show the potential benefits and effectiveness of the developed 
TABLE 4: Maximum allowable time delay bounds $h$ for different values $h_{D}$ in Example 3.

\begin{tabular}{|c|c|c|c|c|c|c|c|c|c|}
\hline$h_{D}$ & 0.1 & 0.2 & 0.3 & 0.4 & 0.5 & 0.6 & 0.7 & 0.8 & 0.9 \\
\hline Theorem 11 & 9.0123 & 8.6789 & 8.4245 & 8.1237 & 7.9028 & 7.4532 & 7.1279 & 6.8325 & 6.0142 \\
\hline
\end{tabular}

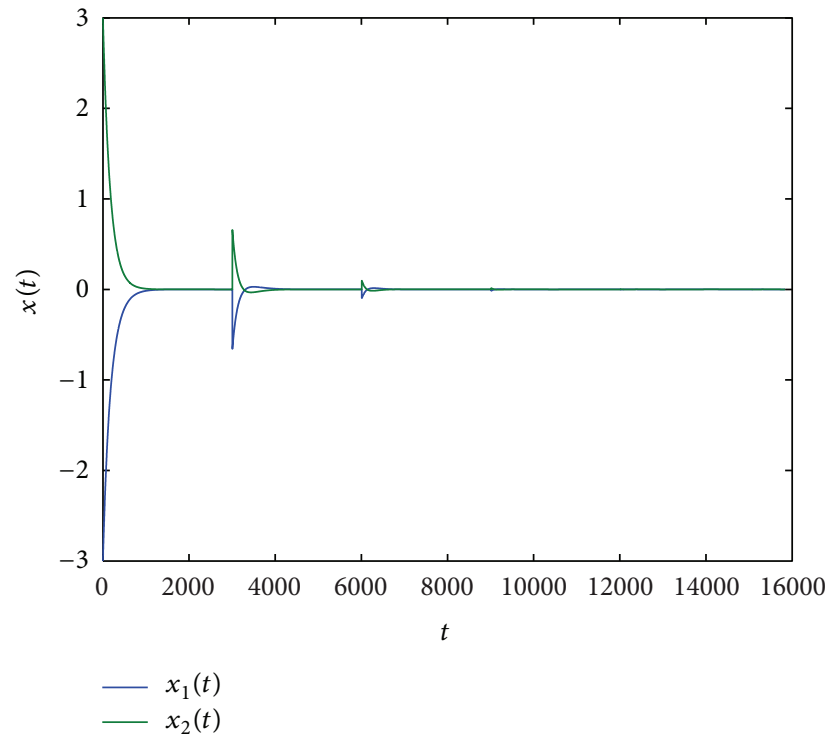

FIgURE 4: The simulation of Example 3 for $h=r=9.0123$, where the initial value is $[-3,3]^{T}, h_{D}=0.1$.

method for delayed neural networks. Consider the delayed system (57) with the following parameters:

Case A

$$
\begin{gathered}
W_{1}=\left[\begin{array}{cccc}
-0.0373 & 0.4852 & -0.3351 & 0.2336 \\
-1.6033 & 0.5988 & -0.3224 & 1.2352 \\
0.3394 & -0.0860 & -0.3824 & -0.5785 \\
-0.1311 & 0.3253 & -0.9534 & -0.5015
\end{array}\right], \\
W_{2}=\left[\begin{array}{cccc}
0.8674 & -1.2405 & -0.5325 & 0.0220 \\
0.0474 & -0.9164 & 0.0360 & 0.9816 \\
1.8495 & 2.6117 & -0.3788 & 0.8428 \\
-2.0413 & 0.5179 & 1.1734 & -0.2775
\end{array}\right], \\
W_{0}=\operatorname{diag}\{1.2769,0.6231,0.9230,0.4480\}, \\
\bar{\Sigma}=\operatorname{diag}\{0.1137,0.1279,0.7994,0.2368\}, \\
\underline{\Sigma}=\operatorname{diag}\{0,0,0,0\} .
\end{gathered}
$$

Case B

$$
\begin{gathered}
W_{1}=\left[\begin{array}{ll}
0.0530 & 0.0454 \\
0.0987 & 0.2750
\end{array}\right], \\
W_{2}=\left[\begin{array}{ll}
0.2381 & 0.9320 \\
0.0388 & 0.5062
\end{array}\right], \\
W_{0}=\operatorname{diag}\{1.5,0.7\}, \quad \bar{\Sigma}=\operatorname{diag}\{0.3,0.8\}, \\
\underline{\Sigma}=\operatorname{diag}\{0,0\} .
\end{gathered}
$$

TABLE 5: Maximum allowable upper bounds $h$ for different $h_{D}$ in Case A.

\begin{tabular}{lccc}
\hline$h_{D}$ & 0.1 & 0.5 & 0.9 \\
\hline$[36]$ & 3.27 & 2.15 & 1.31 \\
{$[37]$} & 3.27 & 2.22 & 1.58 \\
{$[38]$} & 3.30 & 2.53 & 2.08 \\
{$[47]$} & 3.35 & 2.59 & 2.13 \\
{$[48]$} & 3.75 & 2.73 & 2.27 \\
{$[49]$} & 3.70 & 3.12 & 2.59 \\
{$[50]$} & 3.91 & 2.79 & 2.33 \\
{$[42]$} & 4.21 & 3.15 & 2.91 \\
Theorem 15 & 5.19 & 3.92 & 3.36 \\
\hline
\end{tabular}

TABLE 6: Maximum allowable time-delay upper bounds $h$ in Case B.

\begin{tabular}{lcccc}
\hline$h_{D}=0$ & 0.4 & 0.45 & 0.5 & 0.55 \\
\hline$[36]$ & 3.99 & 3.27 & 3.05 & 2.98 \\
{$[48]$} & 4.38 & 3.60 & 3.33 & 3.23 \\
{$[49](m=2)$} & 4.39 & 3.67 & 3.46 & 3.41 \\
{$[19]$} & 4.4801 & 4.0626 & 3.8083 & 3.7064 \\
{$[18](m=2)$} & 5.2420 & 4.4301 & 4.1055 & 3.9231 \\
Theorem 1 in [51] & 5.0588 & 4.2603 & 4.0604 & 4.0185 \\
Theorem 2 in [51] & 5.3079 & 4.5267 & 4.2924 & 4.1903 \\
Corollary 1 in [51] & 9.7094 & 7.7523 & 6.8570 & 6.2977 \\
Theorem 15 & 10.2358 & 8.8378 & 7.9531 & 7.4532 \\
\hline
\end{tabular}

Case C

$$
\begin{gathered}
W_{1}=\left[\begin{array}{cc}
1 & 1 \\
-1 & -1
\end{array}\right], \quad W_{2}=\left[\begin{array}{cc}
0.88 & 1 \\
1 & 1
\end{array}\right], \\
W_{0}=\{2,2\}, \quad \bar{\Sigma}=\operatorname{diag}\{0.4,0.8\}, \\
\underline{\Sigma}=\operatorname{diag}\{0,0\} .
\end{gathered}
$$

From Tables 5, 6, and 7, it is clearly shown that Theorem 15 is less conservative than those in the mentioned literature. In Case $\mathrm{A}$, when $h_{D}=0.1$, the simulation result is given as Figure 5, which implies that under the given conditions, the state vector $x(t)$ stabilizes to zero asymptotically. Hence, this example indicates fully that the method proposed in the paper plays a major role in reducing conservatism.

Finally, we provide the number of decision variables involved in the LIM in each of the Theorems 6-15 in Table 8 .

\section{Conclusions}

This paper is concerned with the stability analysis of neutral type neural networks with mixed time-varying delays. Some improved delay-dependent stability results are established by 
TABLE 7: Maximum allowable time-delay bounds $h$ for different values $h_{D}$ in Case C.

\begin{tabular}{lcccccc}
\hline Method & {$[52]$} & {$[48]$} & {$[53]$} & {$[42]$} & {$[54]$} & Theorem 15 \\
\hline$h_{D}=0.8$ & 2.3571 & 2.8854 & 2.9144 & 3.1409 & 4.1626 & 6.9785 \\
$h_{D}=0.9$ & 1.6050 & 1.9631 & 1.9095 & 1.6375 & 3.9766 & 6.3457 \\
\hline
\end{tabular}

TABLE 8: Number of decision variables involved in the stability criteria.

\begin{tabular}{lllr}
\hline Method & Number of decision variables & Method & Number of decision variables \\
\hline Theorem 6 & {$\left[(12+l)^{2}+8(12+l)+6\right] n^{2}+(16+l) n$} & Theorem 11 & $215 n^{2}+15 n$ \\
Theorem 13 & {$\left[(10+l)^{2}+8(10+l)+6\right] n^{2}+(14+l) n$} & Theorem 15 & $159 n^{2}+13 n$ \\
\hline
\end{tabular}

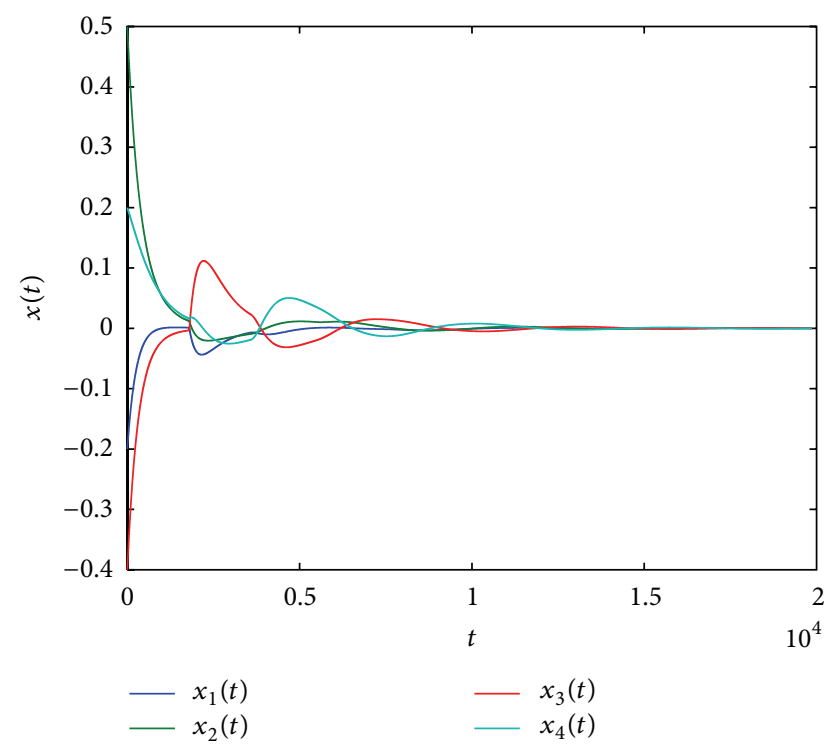

FIgURE 5: The simulation of Example 4 for $h=5.19$, where the initial value is $[-0.2,0.5,-0.4,0.2]^{T}, h_{D}=0.1$.

using a novel approach for the networks. Improved delaydependent stability criteria in terms of linear matrix inequalities (LMIs) are derived by employing a new type of LyapunovKrasovskii functionals with three and four integral terms. Different from previous results by using the first-order convex combination property, our derivation applies the idea of second-order convex combination and the property of quadratic convex function. obtained results are formulated in terms of linear matrix inequalities (LMIs). Numerical examples are given to illustrate the effectiveness and the advantage of the proposed main results.

\section{Acknowledgments}

This work was supported by the National Basic Research Program of China (2010CB732501), the National Natural Science Foundation of China (61273015), and the Natural Science Foundation of Hainan province (111002).

\section{References}

[1] A. Cichocki and R. Unbehauen, Neural Networks for Optimization and Signal Processing, John Wiley \& Sons, Chichester, UK, 1993.
[2] F. Beaufays, Y. Abdel-Magid, and B. Widrow, "Application of neural networks to load-frequency control in power systems," Neural Networks, vol. 7, no. 1, pp. 183-194, 1994.

[3] M. Galicki, H. Witte, J. Dörschel, M. Eiselt, and G. Griessbach, "Common optimization of adaptive preprocessing units and a neural network during the learning period. Application in EEG pattern recognition," Neural Networks, vol. 10, no. 6, pp. 1153$1163,1997$.

[4] L. O. Chua and L. Yang, "Cellular neural networks: applications," IEEE Transactions on Circuits and Systems, vol. 35, no. 10, pp. 1273-1290, 1988.

[5] B. K. Wong and Y. Selvi, "Neural network applications in finance: a review and analysis of literature (1990-1996)," Information and Management, vol. 34, no. 3, pp. 129-139, 1998.

[6] M. Zhenjiang and Y. Baozong, "Analysis and optimal design of continuous neural networks with applications to associative memory," Neural Networks, vol. 12, no. 2, pp. 259-271, 1999.

[7] Z. Waszczyszyn and L. Ziemiański, "Neural networks in mechanics of structures and materials-new results and prospects of applications," Computers and Structures, vol. 79, no. 22-25, pp. 2261-2276, 2001.

[8] A. Rawat, R. N. Yadav, and S. C. Shrivastava, "Neural network applications in smart antenna arrays: a review," International Journal of Electronics and Communications, vol. 66, pp. 903-912, 2012.

[9] S. Fang, M. Jiang, and X. Wang, "Exponential convergence estimates for neural networks with discrete and distributed delays," Nonlinear Analysis: Real World Applications, vol. 10, no. 2, pp. 702-714, 2009.

[10] S. Xu, J. Lam, and D. W. C. Ho, "Novel global robust stability criteria for interval neural networks with multiple time-varying delays," Physics Letters A, vol. 342, no. 4, pp. 322-330, 2005.

[11] Y. Liu, Z. Wang, and X. Liu, "Global exponential stability of generalized recurrent neural networks with discrete and distributed delays," Neural Networks, vol. 19, no. 5, pp. 667-675, 2006.

[12] W. Qian, T. Li, S. Cong, and S. Fei, "Improved stability analysis on delayed neural networks with linear fractional uncertainties," Applied Mathematics and Computation, vol. 217, no. 7, pp. 3596-3606, 2010.

[13] O. Faydasicok and S. Arik, "Robust stability analysis of a class of neural networks with discrete time delays," Neural Networks, vol. 29-30, pp. 52-59, 2012.

[14] N. Mahdavi and M. B. Menhaj, "A new set of sufficient conditions based on coupling parameters for synchronization of Hopfield like Chaotic Neural Networks," International Journal of Control, Automation and Systems, vol. 9, no. 1, pp. 104-111, 2011. 
[15] J. Lian and K. Zhang, "Exponential stability for switched CohenGrossberg neural networks with average dwell time," Nonlinear Dynamics, vol. 63, no. 3, pp. 331-343, 2011.

[16] Z. Zhang, T. Zhang, S. Huang, and P. Xiao, "New global exponential stability result to a general Cohen-Grossberg neural networks with multiple delays," Nonlinear Dynamics, vol. 67, no. 4, pp. 2419-2432, 2012.

[17] O. M. Kwon, J. H. Park, S. M. Lee, and E. J. Cha, "A new augmented Lyapunov-Krasovskii functional approach to exponential passivity for neural networks with time-varying delays," Applied Mathematics and Computation, vol. 217, no. 24, pp. 10231-10238, 2011.

[18] J. Tian and S. Zhong, "Improved delay-dependent stability criterion for neural networks with time-varying delay," Applied Mathematics and Computation, vol. 217, no. 24, pp. 10278-10288, 2011.

[19] O. M. Kwon, J. W. Kwon, and S. H. Kim, "New results on stability criteria for neural networks with time-varying delays," Chinese Physics B, vol. 20, no. 5, Article ID 050505, 2011.

[20] O. M. Kwon, S. M. Lee, and J. H. Park, "On improved passivity criteria of uncertain neural networks with time-varying delays," Nonlinear Dynamics, vol. 67, no. 2, pp. 1261-1271, 2012.

[21] Y. Chen, S. Fei, and K. Zhang, "Improved asymptotic stability conditions for neural networks with discrete and distributed delays," International Journal of Computer Mathematics, vol. 89, no. 15, pp. 1938-1951, 2012.

[22] H. J. Cho and J. H. Park, "Novel delay-dependent robust stability criterion of delayed cellular neural networks," Chaos, Solitons and Fractals, vol. 32, no. 3, pp. 1194-1200, 2007.

[23] C.-H. Lien, K.-W. Yu, Y.-F. Lin, Y.-J. Chung, and L.-Y. Chung, "Global exponential stability for uncertain delayed neural networks of neutral type with mixed time delays," IEEE Transactions on Systems, Man, and Cybernetics B, vol. 38, no. 3, pp. 709720, 2008.

[24] L. Cheng, Z.-G. Hou, and M. Tan, "A neutral-type delayed projection neural network for solving nonlinear variational inequalities," IEEE Transactions on Circuits and Systems II, vol. 55, no. 8, pp. 806-810, 2008.

[25] J. H. Park and O. M. Kwon, "Synchronization of neural networks of neutral type with stochastic perturbation," Modern Physics Letters B, vol. 23, no. 14, pp. 1743-1751, 2009.

[26] P. Balasubramaniam, V. Vembarasan, and R. Rakkiyappan, "Global robust asymptotic stability analysis of uncertain switched Hopfield neural networks with time delay in the leakage term," Neural Computing and Applications, vol. 21, pp. 1593-1616, 2012.

[27] P. Balasubramaniam and V. Vembarasan, "Synchronization of recurrent neural networks with mixed time-delays via output coupling with delayed feedback," Nonlinear Dynamics, vol. 70, no. 1, pp. 677-691, 2012.

[28] S. Lakshmanan, V. Vembarasan, and P. Balasubramaniam, "Delay decomposition approach to state estimation of neural networks with mixed time-varying delays and Markovian jumping parameters," Mathematical Methods in the Applied Sciences, vol. 36, no. 4, pp. 395-412, 2013.

[29] P. Balasubramaniam and V. Vembarasan, "Asymptotic stability of BAM neural networks of neutral-type with impulsive effects and time delay in the leakage term," International Journal of Computer Mathematics, vol. 88, no. 15, pp. 3271-3291, 2011.

[30] P. Balasubramaniam, V. Vembarasan, and R. Rakkiyappan, "Leakage delays in T-S fuzzy cellular neural networks," Neural Processing Letters, vol. 33, no. 2, pp. 111-136, 2011.
[31] X. Li and J. Cao, "Delay-dependent stability of neural networks of neutral type with time delay in the leakage term," Nonlinearity, vol. 23, no. 7, pp. 1709-1726, 2010.

[32] J.-E. Feng, S. Xu, and Y. Zou, "Delay-dependent stability of neutral type neural networks with distributed delays," Neurocomputing, vol. 72, no. 10-12, pp. 2576-2580, 2009.

[33] Z. Orman, "New sufficient conditions for global stability of neutral-type neural networks with time delays," Neurocomputing, vol. 97, pp. 141-148, 2012.

[34] S. Lakshmanan and P. Balasubramaniam, "New results of robust stability analysis for neutral-type neural networks with timevarying delays and Markovian jumping parameters," Canadian Journal of Physics, vol. 89, no. 8, pp. 827-840, 2011.

[35] Z. Feng and J. Lam, "Integral partitioning approach to robust stabilization for uncertain distributed time-delay systems," International Journal of Robust and Nonlinear Control, vol. 22, no. 6, pp. 676-689, 2012.

[36] C. C. Hua, C. N. Long, and X. P. Guan, "New results on stability analysis of neural networks with time-varying delays," Physics Letters A, vol. 352, no. 4-5, pp. 335-340, 2006.

[37] Y. He, G. P. Liu, and D. Rees, "New delay-dependent stability criteria for neural networks with time-varying delay," IEEE Transactions on Neural Networks, vol. 18, no. 1, pp. 310-314, 2007.

[38] Y. He, G. P. Liu, D. Rees, and M. Wu, "Stability analysis for neural networks with time-varying interval delay," Neural Networks, vol. 18, no. 6, pp. 1850-1854, 2007.

[39] S. Lakshmanan, H. Ju. Park, H. Y. Jung, O. M. Kwon, and R. Rakkiyappan, "A delay partitioning approach to delaydependent stability analysis for neutral type neural networks with discrete and distributed delays," Neurocomputing, vol. 111, pp. 81-89, 2013.

[40] S. Boyd, L. El Ghaoui, E. Feron, and V. Balakrishnan, Linear Matrix Inequalities in System and Control Theory, vol. 15, Society for Industrial and Applied Mathematics, Philadelphia, Pa, USA, 1994.

[41] J.-H. Kim, "Note on stability of linear systems with time-varying delay," Automatica A, vol. 47, no. 9, pp. 2118-2121, 2011.

[42] H. G. Zhang, F. S. Yang, X. D. Liu, and Q. J. Zhang, "Stability analysis for neural networks with time-varying delay based on quadratic convex combination," Transctions on Neural Networks and Learning Systems, vol. 24, pp. 513-521, 2013.

[43] K. Gu, "An integral inequality in the stability problem of timedelay systems," in Proceedings of the 39th IEEE Conference Decision Control, pp. 2805-2810, Sydney, Australia, 2000.

[44] P. Balasubramaniam, R. Krishnasamy, and R. Rakkiyappan, "Delay-dependent stability of neutral systems with timevarying delays using delay-decomposition approach," Applied Mathematical Modelling, vol. 36, no. 5, pp. 2253-2261, 2012.

[45] X. Zhu and Y. Wang, "Delay-dependent exponential stability for neural networks with discrete and distributed time-varying delays," Physics Letters A, vol. 373, no. 44, pp. 4066-4072, 2009.

[46] J. Tian and S. Zhong, "New delay-dependent exponential stability criteria for neural networks with discrete and distributed time-varying delays," Neurocomputing, vol. 74, no. 17, pp. 33653375, 2011.

[47] H. G. Zhang, Z. Liu, G.-B. Huang, and Z. Wang, "Novel weighting-delay-based stability criteria for recurrent neural networks with time-varying delay," IEEE Transactions on Neural Networks, vol. 21, no. 1, pp. 91-106, 2010.

[48] O. M. Kwon and J. H. Park, "Improved delay-dependent stability criterion for neural networks with time-varying delays," Physics Letters A, vol. 373, no. 5, pp. 529-535, 2009. 
[49] J. Sun, G. P. Liu, and J. Chen, "Delay-dependent stability and stabilization of neutral time-delay systems," International Journal of Robust and Nonlinear Control, vol. 19, no. 12, pp. 13641375, 2009.

[50] J. Tian and X. Xie, "New asymptotic stability criteria for neural networks with time-varying delay," Physics Letters A, vol. 374, no. 7, pp. 938-943, 2010.

[51] O. M. Kwon, M. J. Park, S. M. Lee, J. H. Park, and E. J. Cha, "Stability for neural networks with time-varying delays via some new approaches," IEEE Transactin on Neutal Networks and Learning Systems, vol. 24, pp. 181-193, 2013.

[52] Q. K. Song, "Exponential stability of recurrent neural networks with both time-varying delays and general activation functions via LMI approach," Neurocomputing, vol. 71, no. 13-15, pp. 28232830, 2008.

[53] Y. Li, J. Tian, J. Zhao, L. Zhang, and T. Li, "Improved stability analysis for neural networks with time-varying delay," Mathematical Problems in Engineering, vol. 2012, Article ID 950269, 19 pages, 2012.

[54] P. L. Liu, "Improved delay-dependent robust stability criteria for recurrent neural networks with time-varying delaying," ISA Transanction, vol. 52, pp. 30-35, 2013. 


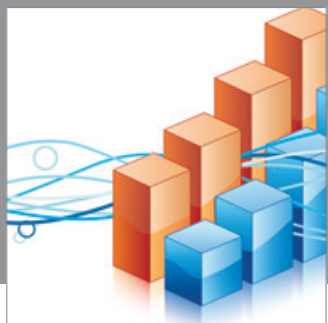

Advances in

Operations Research

mansans

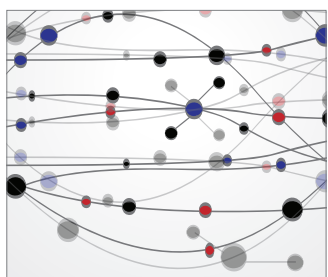

The Scientific World Journal
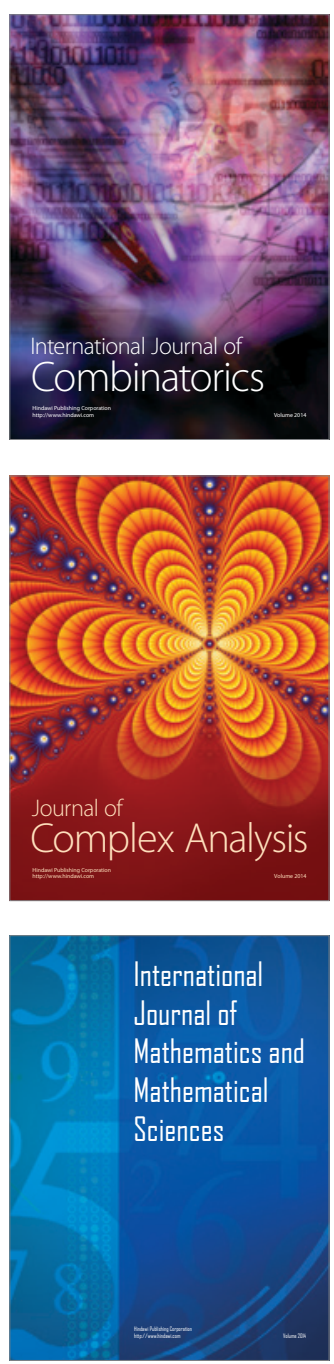
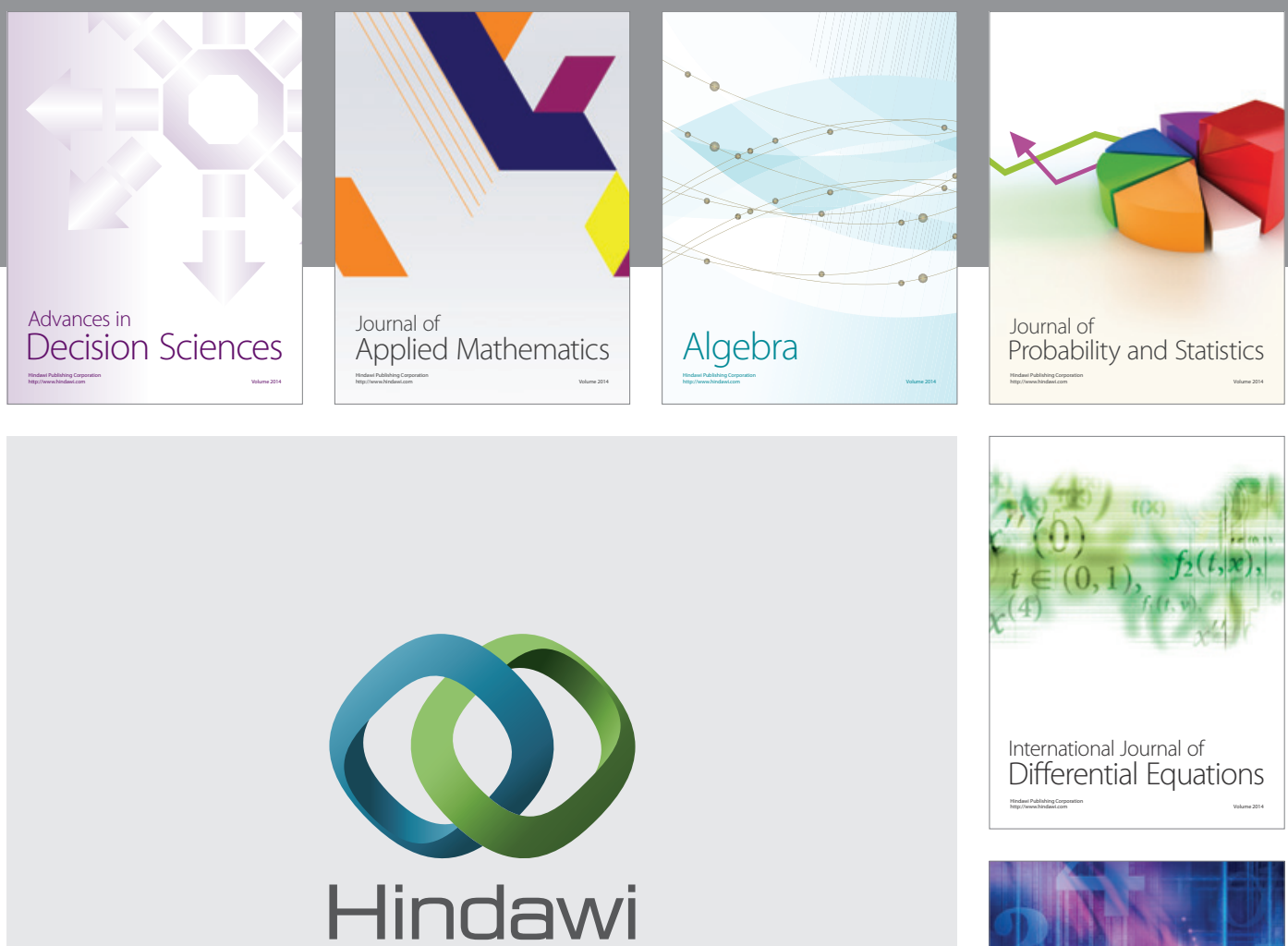

Submit your manuscripts at http://www.hindawi.com
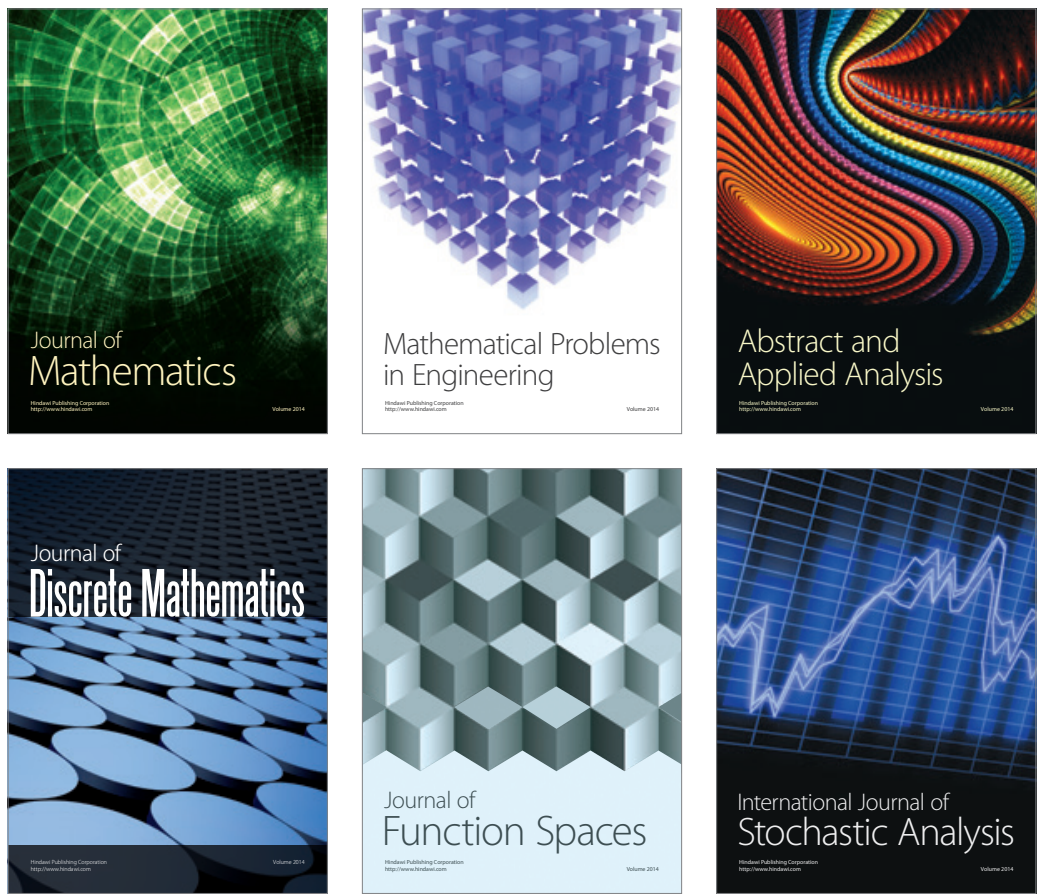

Journal of

Function Spaces

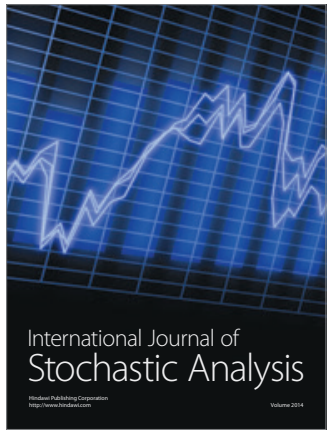

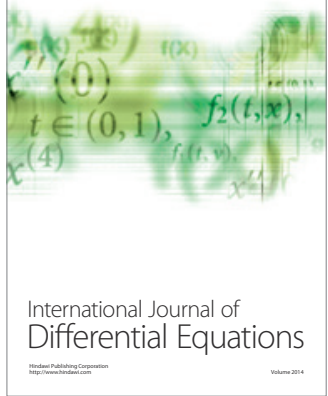
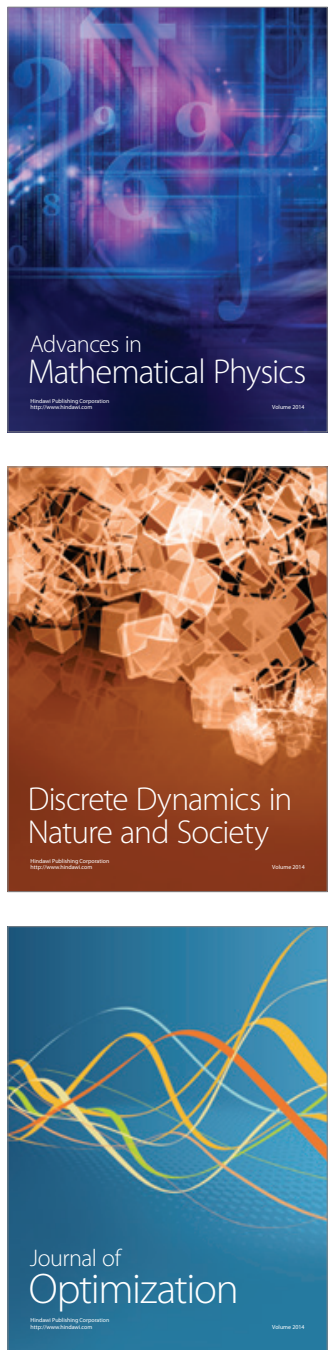